\title{
Multiple Targeted Self-Emulsifying Compound RGO Reveals Obvious Antitumor Potential in Hepatocellular Carcinoma
}

\section{Sanxiu He}

The First Affiliated Hospital of Chongqing Medical University

Shaorong Tian

The First Affiliated Hospital of Chongqing Medical University

Xin Le

The First Affiliated Hospital of Chongqing Medical University

\section{Jialin Chen}

Beijing University of Chinese Medicine Affiliated Chongqing Traditional Chinese Medicine Hospital:

Chongqing City Hospital of Traditional Chinese Medicine

\section{Hongyi Chen}

Beijing University of Chinese Medicine Affiliated Chongqing Traditional Chinese Medicine Hospital:

Chongqing City Hospital of Traditional Chinese Medicine

\section{Xiaoqian He}

The First Affiliated Hospital of Chongqing Medical University

Junhao Mu

The First Affiliated Hospital of Chongqing Medical University

\section{$\mathrm{Ke} \mathrm{Xu}$}

The First Affiliated Hospital of Chongqing Medical University

\section{Qin Xiang}

The First Affiliated Hospital of Chongqing Medical University

\section{Yue Wu}

The First Affiliated Hospital of Chongqing Medical University

\section{Yijiao Ning}

The First Affiliated Hospital of Chongqing Medical University

\section{Jiong Chen}

Beijing University of Chinese Medicine Affiliated Chongqing Traditional Chinese Medicine Hospital: Chongqing City Hospital of Traditional Chinese Medicine

\section{Tingxiu Xiang ( $\square$ larissaxiang@163.com )}

Chongqing Key Laboratory of Molecular Oncology and Epigenetics, the First Affiliated Hospital of Chongqing Medical University 
Research

Keywords: hepatocellular carcinoma, ginsenoside Rg3, ganoderma lucidum polysaccharide, Oridonin, epidermal growth factor receptor

Posted Date: December 29th, 2020

DOl: https://doi.org/10.21203/rs.3.rs-135610/v1

License: (c) (i) This work is licensed under a Creative Commons Attribution 4.0 International License.

Read Full License 


\section{Abstract}

Background: Currently, the treatment for advanced hepatocellular carcinoma (HCC) is extremely limited. Ginsenoside Rg3, ganoderma lucidum polysaccharide, and Oridonin have shown great potential in antitumor therapy in previous studies, but low bioavailability and poor solubility seriously hinder their clinical application. Hence, new strategy for liver cancer are urgently needed.

Methods: Cell viability, cell proliferation, cell migration, colony formation, tubule formation, sphere formation, and flow cytometry were used to assess the effect of a new drug, RGO-SMEDDS, (selfmicroemulsifying drug delivery system comprising of $\mathrm{Rg} 3$, ganoderma lucidum polysaccharide, and Oridonin) on HCC. Specific anti-tumor mechanisms of RGO-SMEDDS were investigated by western blot, qRT-PCR, and immunohistochemistry. Xenografts and staining with hematoxylin and eosin were used to assess the effects of RGO-SMEDDS on tumorigenesis in vivo.

Results: We developed a self-microemulsifying drug delivery system (RGO-SMEDDS) for these three plant monomers. Treatment with RGO-SMEDDS resulted in induction of G2/M phase arrest and apoptosis, inhibition of migration and invasion, and suppression of cell proliferation, both in vitro and in vivo. Furthermore, RGO-SMEDDS restored immune function by suppressing the production of immunosuppressive cytokine and M2-polarized macrophages, reduced angiogenesis by down-regulation of vascular endothelial growth factor and its receptor, and attenuated stemness of HCC by inhibiting EGFR/AKT/GSK-3a/ $\beta$ signaling pathways. In addition to excellent anti-tumor effects, RGO-SMEDDS showed considerable safety in acute toxicity tests.

Conclusion: RGO-SMEDDS exerted significant anti-tumor effects by reducing angiogenesis, remodeling immune microenvironments, and promoting apoptosis, without obvious toxicities. With these attributes RGO-SMEDDS is a promising therapy for the treatment of hepatocellular carcinoma.

\section{Background}

Hepatocellular carcinoma (HCC) is one of the most common and deadliest cancers worldwide, with a faster incidence than other cancers [1, 2]. For patients in early stage of HCC, surgical resection is currently the most favorable treatment. However, for patients with advanced HCC the current systemic therapies using molecular-targeted drugs remain unsatisfactory, mainly due to drug resistance $[3,4]$. The 5 -year overall survival rate is less than $10 \%$, which necessitates the development of new HCC treatment strategies $[3,5]$. Cancer is one of the most devastating diseases, involving multiple genetic changes and cell abnormalities. Monotherapies are often unable to comprehensively and effectively inhibit tumor growth $[6,7]$. Multidrug therapy is advantageous due to the effect on multiple signaling pathways simultaneously. The resultant anti-tumor effects can more effectively reduce drug resistance [6, 8-11]. In this study, we evaluated the anti-tumor effect and mechanism of a new self-emulsifying compound, RGOSMEDDS. Results showed RGO-SMEDDS likely to be a potential new drug for treatment of liver cancer. 
$\mathrm{HCC}$ have been evidenced to evade immune attack by multiple mechanisms including absence of tumor antigens [12-14], secretion of suppressive molecules [15], expression of inhibitory checkpoint molecules [16-18], and the recruitment of immunosuppressive cell populations $[19,20]$. These mechanisms impede cellular immune function, abrogate the anti-tumor immune response, and result in an immune tolerant microenvironment. Given the inflammatory background of HCC, the hepatic tumor microenvironment (TME) plays a critical role in tumor progression, recurrence, and metastasis. Therefore, the therapy focusing on the modulation of TME are particularly promising. Ganoderma lucidum polysaccharides (GLP) is the main bioactive component of a water soluble extract of the mushroom Ganoderma lucidum [21]. GLP exerts anticancer effects, in part, by immune function augmentation including activating immune cells, promoting lymphocyte proliferation, enhancing phagocytosis, increasing cytokine production, and enhancing NK cell-mediated cytotoxicity [22-24]. Hence, GLP has the potential to improve the tumor-immune microenvironment.

Many cancers tend to induce angiogenesis through overexpression of vascular endothelial growth factor and its receptor (VEGF/VEGFR) or by shaping the hypoxic tumor microenvironment [25]. HCC is a hypervascular tumor in which angiogenesis plays a important role in tumor growth and spread. Hence, anti-angiogenic treatment remains the main method in systemic therapy of HCC [26, 27]. The 20(S)ginsenoside-Rg3, a natural triterpenoid saponin extracted from red ginseng, has been shown to possess significant anticancer activity by the inhibition of migration and angiogenesis [28, 35]. Rg3 treatment can inhibit hypoxia-induced expression of HIF-1, COX-2, and VEGF by targeting a variety of signaling pathways, such as STAT3, ERK1/2, NFkB, and JNK [30-32], showing strong anti-angiogenic capacity.

In addition to immune avoidance and angiogenesis promotion, $\mathrm{HCC}$ also stimulate proliferation-related signaling pathways and as well inhibit apoptosis-related signaling pathways to guarantee rapid tumor proliferation. Inhibition of cancer cell proliferation and induction of cancer cell death are effective strategies in the treatment of liver cancer [33]. Oridonin, a diterpenoid isolated from the medicinal herb Rabdosia rubescens, has been proven carrying remarkable anti-tumor effects [33,34]. A large number of studies have demonstrated cell cycle arrest and apoptosis induction of various malignant cancer cells by Oridonin. These effects are due to the regulation of intracellular reactive oxygen species (ROS), Bcl-2/Bax, NF-KB, p53/p21, MAPK, PI3K, microRNA, and fatty acid synthase pathways [35-40]. Although Oridonin appears to be effective for cancer treatment, its underlying mechanism is very complicated and its direct target remains disclosure [33].

SMEDDS is an isotropic transparent or semi-transparent dispersion system composed of oil phase, emulsifier and co-emulsifier, which is characterized by the automatic formation of oil-in-water $(0 / W)$ type microemulsion under physiological conditions such as body temperature and gastrointestinal perimosis. SMEDDS has been shown to improve the solubility and bioavailability of insoluble and fat-soluble drugs, increase absorption rates and stability, and overcome first-pass effects through lymphatic circulation[41, 42]. In addition, its industrial preparation and administration are convenient. Although GLP, Rg3, and Oridonin have impressive anti-tumor effects, their clinical application is limited due to low solubility and poor bioavailability $[34,35]$. To resolve these problems, we constructed a new drug, RGO-SMEDDS, which 
is a self-emulsifying compound comprising of Rg3, GLP, and Oridonin (sFig. 1). Our results demonstrated RGO-SMEDDS to be anti-angiogenic, to inhibit M2 macrophages, and promote apoptosis. Further, RGOSMEDDS may be an EGFR inhibitor that reduced the stemness of HCC through the PI3K/AKT/GSK-3a/ $\beta$ signaling pathway .

\section{Materials And Methods}

\section{Chemicals}

Ginsenoside Rg3, ganoderma lucidum polysaccharide and Oridonin used in this study was obtained from Nanjing Zelang Medical Technology Co. Ltd (China). Soybean lecithin was a product of Tianjin kwangfu Fine Chemical Industry Research Institute (China). Ethyl oleate were purchased from Shandong Jining Hongming Chemical Reagent Co (China) and span-80 was purchased from Chengdu Kelong Chemical Reagent Factory (China).

\section{Preparation of RGO-SMEDDS}

$6 \mathrm{mg} / \mathrm{ml}$ Ginsenoside Rg3, $20 \mathrm{mg} / \mathrm{ml}$ ganoderma lucidum polysaccharide and $20 \mathrm{mg} / \mathrm{ml}$ Oridonin were added into the mixture of tetrahydrofuran and $50 \mathrm{mg} / \mathrm{ml}$ soybean lecithin and stirred at $55 \sim 65^{\circ} \mathrm{C}$ at a constant speed until completely dissolved. The lecithin complex were formed after the removal of tetrahydrofuran by rotary evaporation. With ethyl oleate as the oil phase, Tween 80 as the emulsifier, and polyethylene glycol-400 as the solvent, a ternary phase diagram was used to construct the selfemulsifying system of RGO. The lecithin complex was added to the mixed system of oil phase, emulsifier and solvent in a ratio of 2:5:2. The mixture was then stirred at $37 \sim 45^{\circ} \mathrm{C}$ and $100 \sim 500 \mathrm{r} / \mathrm{min}$ until a light blue transparent liquid was formed. (Patent No : 2020ZY013799)

\section{Cell lines}

Cell lines (HepG2, Huh7, Hep3B, LO2, and THP-1) were obtained from the American Type Culture Collection (ATCC, Manassas, VA, USA) or collaborators. Cells were cultured in RPMI 1640 medium (GibcoBRL, Karlsruhe, Germany) supplemented with $10 \%$ fetal bovine serum (FBS) plus $1 \%$ penicillin and streptomycin at $37^{\circ} \mathrm{C} / 5 \% \mathrm{CO}_{2}$, as advised by ATCC.

\section{RNA isolation, reverse transcription (RT)-PCR, and real-Time PCR}

Total RNA isolation was achieved by using TRIzol Reagent ${ }^{\circledR}$ (Molecular Research Center, Cincinnati, OH, USA) and the manufacturer's instructions. Reverse transcriptase-polymerase chain reaction (RT-PCR) was performed to generate cDNA in a total volume of $20 \mu \mathrm{L}$. RT-PCR was performed using Go-Taq (Promega, Madison, $\mathrm{WI}, \mathrm{USA}$ ) with the following conditions; initial denaturation at $95^{\circ} \mathrm{C}$ for $2 \mathrm{~min}, 32$ amplification cycles (denaturation: $95^{\circ} \mathrm{C}$ for $30 \mathrm{~s}$ : annealing: $55^{\circ} \mathrm{C}$ for $30 \mathrm{~s}$ : and extension: $72^{\circ} \mathrm{C}$ for $30 \mathrm{~s}$ ), with final extension at $72^{\circ} \mathrm{C}$ for $3 \mathrm{~min}$. $\beta$-actin was used as an internal control with only 23 cycles for amplification. RT-PCR was accomplished with a SYBR Green kit (Thermo Fisher) and a 7500 Real-Time PCR System 
(Applied Biosystems, Foster City, CA, USA). Specific primer sequences are listed in Table 1, with p-values for each group determined by Student's $t$-test. Each sample was assessed in three independent experiments. 
Table 1

List of primers used in this study

\begin{tabular}{|c|c|c|c|c|}
\hline PCR & Primer & Sequence $\left(5^{\prime}-3^{\prime}\right)$ & $\begin{array}{l}\text { Product size } \\
\text { (bp) }\end{array}$ & $\begin{array}{l}\text { Annealing temperature } \\
\left({ }^{\circ} \mathrm{C}\right)\end{array}$ \\
\hline qRT- & IL2F & TACAAGAACCCGAAACTGACTCG & $223 \mathrm{bp}$ & 60 \\
\hline \multirow[t]{25}{*}{ PCR } & $I L 2 R$ & ACATGAAGGTAGTCTCACTGCC & & \\
\hline & IL4F & ССААСТGСТTСССССТСТG & $150 \mathrm{bp}$ & 60 \\
\hline & IL4R & TCTGTTACGGTCAACTCGGTG & & \\
\hline & IL $10 F$ & GACTTTAAGGGTTACCTGGGTTG & 112 bp & 60 \\
\hline & IL 10R & TCACATGCGCCTTGATGTCTG & & \\
\hline & IL $13 F$ & GAAGGCTCCGCTCTGCAAT & 75 bp & 60 \\
\hline & IL 13R & TCCAGGGCTGCACAGTACA & & \\
\hline & M-CSFF & TGGCGAGCAGGAGTATCAC & 108 bp & 60 \\
\hline & M-CSFR & AGGTCTCCATCTGACTGTCAAT & & \\
\hline & $C D 163 F$ & TTTGTCAACTTGAGTCCCTTCAC & $127 \mathrm{bp}$ & 60 \\
\hline & $C D 163 R$ & TCCCGCTACACTTGTTTTCAC & & \\
\hline & $A R G 1 F$ & GTGGAAACTTGCATGGACAAC & 76 bp & 60 \\
\hline & $A R G 1 R$ & AATCCTGGCACATCGGGAATC & & \\
\hline & VEGFAF & AGGGCAGAATCATCACGAAGT & 75 bp & 60 \\
\hline & VEGFAR & AGGGTCTCGATTGGATGGCA & & \\
\hline & VEGFRF & GGCCCAATAATCAGAGTGGCA & $109 \mathrm{bp}$ & 60 \\
\hline & VEGFRR & CCAGTGTCATTTCCGATCACTTT & & \\
\hline & SOX $2 F$ & GCCGAGTGGAAACTTTTGTCG & $155 \mathrm{bp}$ & 60 \\
\hline & $S O X 2 R$ & GGCAGCGTGTACTTATCCTTCT & & \\
\hline & NANOGF & TTTGTGGGCCTGAAGAAAACT & 116 bp & 60 \\
\hline & NANOGR & AGGGCTGTCCTGAATAAGCAG & & \\
\hline & $C D 133 F$ & AGTCGGAAACTGGCAGATAGC & 99 bp & 60 \\
\hline & $C D 133 R$ & GGTAGTGTTGTACTGGGCCAAT & & \\
\hline & OCT3/4F & CTGGGTTGATCCTCGGACCT & $243 \mathrm{bp}$ & 60 \\
\hline & OCT3/4R & CCATCGGAGTTGCTCTCCA & & \\
\hline
\end{tabular}




\begin{tabular}{|c|c|c|c|c|}
\hline PCR & Primer & Sequence $\left(5^{\prime}-3^{\prime}\right)$ & $\begin{array}{l}\text { Product size } \\
\text { (bp) }\end{array}$ & $\begin{array}{l}\text { Annealing temperature } \\
\left({ }^{\circ} \mathrm{C}\right)\end{array}$ \\
\hline & $c-M y c F$ & GGCTCCTGGCAAAAGGTCA & 119 bp & 60 \\
\hline & $c-M y c R$ & CTGCGTAGTTGTGCTGATGT & & \\
\hline & $\beta$-actinF & GTCTTCCССTCCATCGTG & 113 bp & 60 \\
\hline & $\beta$-actinR & AGGGTGAGGATGCCTCTCTT & & \\
\hline
\end{tabular}

\section{Cell proliferation and colony formation assays}

HepG2 and Huh7 cells were seeded into 96-well plates at a density of 2,000/well. The effect of RGOSMEDDS was assessed at concentrations of $0,0.15,0.30,0.60$, and $1.20 \mu \mathrm{M}$. Cell proliferation at different time periods ( 0 h, 24, 48, and $72 \mathrm{~h}$ ) was measured by absorbance at $450 \mathrm{~nm}$ with a Cell Counting Kit-8 (CCK-8; Beyotime, Shanghai, China). For the colony formation assay, HepG2 and Huh7 were placed into 6-well plates with increasing concentrations of RGO-SMEDDS (200, 400, 800/well). After 14 days of incubation, cell colonies were stained with Crystal Violet and evaluated by scanning with a CanoScan 8800F MOEL-85 scanner.

\section{Flow cytometry (FCM)}

Cell cycle and apoptotic status were estimated by FCM. For apoptosis, cells were treated with or without RGO-SMEDDS, harvested, and double-stained with propidium iodide (PI) and annexin V-fluorescein isothiocyanate. For cell cycle analysis, cells were collected and fixed with ice-cold $75 \%$ ethanol, treated with RNase, and stained with PI. Three individual samples were assessed for each group.

\section{Nude mouse tumor xenograft model}

This experiments complyed with the ARRIVE guidelines and was carried out in accordance with the U.K. Animals (Scientific Procedures) Act. $1 \times 10^{7} \mathrm{HepG} 2$ cells were subcutaneously injected into nude mice (male, $n=14$ ) and $1 \times 10^{6}$ Hep1- 6 cells into C57BL/ 6 mice (male, $n=20$ ). Mice were randomly divided into control and treated groups on the fifth day after transplantation. The treated group was given $10 \mathrm{mg} / \mathrm{kg}$ RGO-SMEDDS orally every day for 2 weeks, while the control group was treated with an equal volume of PBS. Tumor size was measured every 2 days. During the next $30 \mathrm{~d}$, a vernier caliper was used to measure tumor size (as judged by length and width) every 3 days. Tumor volume was determined using the formula: volume $=$ length $\times$ width ${ }^{2} \times 0.52$.

\section{Transwell and wound healing assays for cell migration and invasion}

Cell migration was assessed by Transwell and wound healing assays as previously described[43]. Transwell $\circledast$ chambers were purchased from Corning Company (Corning Incorporated, 2 Alfred Road, and Kennebunk ME 04043 USA). For the invasion assay, Matrigel glue (BD Biosciences) was placed on the 
upper surface of the chambers. The cells on the chamber's lower surface were assessed by phasecontrast microscopy (Leica), $24 \mathrm{~h}$ after seeding. All experiments were conducted in triplicate.

\section{Western blot}

Protein lysates $(40 \mu \mathrm{g})$ were separated by sodium dodecyl sulphate-polyacrylamide gel electrophoresis (SDS-PAGE) and transferred to polyvinylidene difluoride (PVDF) membranes. The primary antibodies used were reactive with; VEGF (\#ab1316,Abcam), VEGFR (\#ab11939,Abcam), AKT (\#ab38449,Abcam), P-AKT (\#ab8805; Abcam), GSK3a/ß\#scc-7291, santa cruz biotechnology), pGSK3a/ $\beta$ (\#sc-81496,santa cruz biotechnology), EGFR (sc373746, santa cruz), and P-EGFR (sc-377547; Santa Cruz). Secondary antibodies were used at a dilution of 1:2,000 at room temperature. Protein bands were visualized by Immobilon Western Chemiluminescent HRP Substrate kit (Millipore Corporation, Billerica, MA, USA).

\section{Immunohistochemistry (IHC) staining}

Tissues were fixed in $4 \%$ paraformaldehyde, sent for sectioning, and assessed with Immunohistochemistry Kit (ZSGB-BIO, Beijing, China). Slides were incubated at $4^{\circ} \mathrm{C}$ overnight $(16-20 \mathrm{~h})$ with antibodies reactive with Ki67 (\#ab16667, Abcam), F4/80 (\#ab100790, Abcam), CD163 (\#sc58965, santa cruz), VEGF (\#ab32152, Abcam), VEGFR (\#ab32152, Abcam), CD34 (\#ab81289, Abcam), EGFR (\#sc373746, santa cruz), and P-EGFR (\#sc-377547, Santa Cruz). DAB (K176810E, ZSGB-BIO, China) was used for target protein staining and Hematoxylin and Eosin (HE) for nuclear staining. Images were photographed at $400 \times$ with a microscope.

\section{Tube formation assay}

A tube formation assay was used to assess angiogenesis. Matrigel assay kits (Chemicon, Millipore) were used per the manufacturer's instructions. Matrigel was allowed to solidify inside an incubator for 30 min. Then, 20,000 HUVECs/well were transferred to chilled pellets and further incubated with conditioned medium derived from HCC cells treated with or without RGO-SMEDDS for 6-24 $\mathrm{h}$. To determine angiogenesis, a branching index for each group was determined at the termination of drug usage.

\section{Sphere-forming assay}

Nonadherent dishes (Costar, NY, USA) were used for this assay. Cells were plated and maintained in serum-free medium (SFM) consisting of DMEM-F12 (Gibco, Carlsbad, CA, USA ), $30 \mathrm{ng} / \mathrm{mL}$ epidermal growth factor (Sino Biological, \#10605), $15 \mathrm{ng} / \mathrm{mL}$ basic fibroblast growth factor (Sino Biological, \#10014), $10 \mu \mathrm{g} / \mathrm{mL}$ insulin (Sino Biological, \#11038), 0.4\% BSA (Sigma-Aldrich, St. Louis, MO, USA), and 2\% B27 (Gibco, Carlsbad, CA, USA). Photographs were obtained and the number of spheres assessed by microscopy (Nikon, Tokyo, Japan).

\section{Target prediction of Oridonin}

The 3D structure of Oridonin was obtained from the PubChem database. The PharmMapper server was used for target prediction of Oridonin. The R software was used for the $\mathrm{GO}$ enrichment analyses of the drug targets. $P<0.01$ was set as the threshold and the 10 top-ranked biological processes were shown. 
The PPI analysis of tyrosine kinase-related targets was performed using the string software. Network visualization and analysis are performed using Cytoscape software. The PDB format structure of the EGFR protein (PDB ID: 5GNK) is downloaded from the RCSB database. AutoDock Tools 1.5.6 Software was used for hydrogenation, charge calculation, atom type distribution, etc. Finally, AutoDock Vina 1.1.2 was used for molecular docking, and Discovery Studio 2020 was used for visual analysis.

\section{RGO in vivo toxicity}

This experiments complyed with the ARRIVE guidelines and was carried out in accordance with the U.K. Animals (Scientific Procedures) Act. 40 C57BL/6 mice aged 6-8 weeks were randomly divided into four groups ( $n=10$, half male and half female) and given a single oral dose of $0,100,200$, or $1,000 \mathrm{mg} / \mathrm{kg}$ of RGO. The LD50 assay was performed with the maximum dose $(1,000 \mathrm{mg} / \mathrm{kg})$. Twenty mice were randomly divided into control and treated groups $(n=10)$. The treated group received oral RGO, $1,000 \mathrm{mg} / \mathrm{kg}$, for once. The mental state and activity of the mice were observed daily and their weight measured once a week. All mice were sacrificed 14 days after drug administration. Collectable organs including brain, heart, lungs, stomach, pancreas, liver, kidneys, ovaries, uterus, bowel and spleen were harvested right after sacrifice. Samples were prepared with formalin and embedded with paraffin using a Ventana machine.

\section{Statistical analyses}

All statistical analyses were assessed as means with SPSS software (version 16, SPSS, Chicago, IL, USA). $X^{2}$ and Fisher's exact tests were utilized for $p$ value calculation. Data were considered statistically meaningful at $p<0.05$.

\section{Results}

\section{RGO-SMEDDS inhibits HCC proliferation by inducing apoptosis and G2 phase arrest.}

Functional assays were performed to assess the anti-tumor effects of RGO-SMEDDS. First, we examined the effects of $0,0.15,0.30,0.60$, and $1.20 \mu \mathrm{M}$ RGO-SMEDDS on the proliferation of HCC and LO2 cells by CCK8 assay (Fig. 1A). RGO-SMEDDS inhibited the proliferation of liver cancer cells in a dose-dependent manner and did no harm to normal cells within the prescribed concentration range. In order to determine an appropriate administration concentration, we assessed the semi-lethal dose of RGO-SMEDDS for Huh7 and HepG2 cells by IC50 assay (Fig. 1B). The RGO-SMEDDS IC50s for Huh7 and HepG2 were $1.2 \mu \mathrm{M}$ and $2.0 \mu \mathrm{M}$, respectively. And this dose was used as the treatment concentration for subsequent experiments in vitro. Clone formation was used to assess the effects of RGO-SMEDDS on cell viability. RGO-SMEDDS significantly inhibited the viability of Huh7 and HepG2 cells (Fig. 1C). As judged by FCM, RGO-SMEDDS increased the percentage of cells in the G2/M phase compared to control (Fig. 1D). Furthermore, RGO-SMEDDS induced a higher apoptosis rate as judged by FCM with V-FITC and PI staining (Fig. 1E). Overall, RGO-SMEDDS effectively produced anti-tumor effects by inhibiting cell proliferation, reducing cell viability, inducing cell cycle arrest, and promoting cell apoptosis. 
RGO-SMEDDS suppresses xenograft tumor growth in vivo.

The above experiments demonstrated the anti-tumor effects of RGO-SMEDDS in vitro. To assess the effects of RGO-SMEDDS on HCC in vivo, Subcutaneous xenograft models were constructed in nude mice and C57B/L mice. As shown in (Fig. 2A-F), tumor size of RGO-SMEDDS-treated mice was significantly reduced compared to control mice, indicating significant inhibition of liver cancer growth. Furthermore, more obvious nuclear fragmentation and proliferation inhibition was found in RGO-treated group via HE and IHC staining assay (Fig. 2G).

\section{RGO-SMEDDS inhibits HCC invasion, migration, and angiogenesis.}

The effects of RGO-SMEDDS on metastasis were assessed. By migration assay, RGO-SMEDDS reduced cell migration (Fig. 3A) and by invasion assay, RGO-SMEDDS significantly weakened the ability of HCC cells to invade barriers (Fig. 3B). The effect of RGO-SMEDDS on angiogenesis was measured by tubule formation assay (Fig. 3C). HUEVC cells were cultured in conditioned medium of HepG2 and Huh7 cells treated or not with RGO-SMEDDS for $48 \mathrm{~h}$. RGO-SMEDDS significantly inhibited angiogenesis induced by HepG2 and Huh7 cells. In addition, we performed qRT-PCR and WB analysis to investigate the mechanism of RGO on angiogenesis (Fig. 3D-E). Compared to control, the expression of VEGF and VEGFR in RGOSMEDDS-treated HCC cells and tissues was significantly down-regulated. Similar results were found by IHC, where RGO-SMEDDS significantly down-regulated VEGF and VEGFR levels in tumor tissues (Fig. 3F). Further, the number of blood vessels in the treated group was much less than in control. Taken together, these results demonstrated RGO-SMEDDS can effectively inhibit the migration, invasion, and angiogenesis of HCC cells.

\section{RGO-SMEDDS inhibits the production of immunosuppressive cytokine and M2 macrophages.}

The qRT-PCR was used to assess cytokine mRNA expression levels in HCC cells and tumor tissues treated with or without RGO-SMEDDS(Fig. 4A). RGO-SMEDDS significantly reduced the expression levels of IL2, IL4, IL10, IL13, and M-CSF1, which are closely related to M2 macrophage differentiation. Based on these results we speculated that RGO-SMEDDS inhibited M2-polarized macrophage induced by HCC cells. In order to verify this speculation, we added RGO-SMEDDS to the THP1-HCC co-culture system, and investigated the mRNA expression level of M2 macrophage markers in THP1 cells by qRT-PCR (Fig. 4B). The expression levels of IL-10, CD163, and Arg1 were significantly down-regulated in THP1 cells from RGO-SMEDDS added co-cultures system. Moreover, we found RGO-SMEDDS to directly inhibit IL10, CD163, and Arg1 in THP1 cells, which indicated that RGO-SMEDDS could not only prevent the M2polarized macrophage induced by HCC cells, but also directly inhibit the formation of M2 macrophages. $\mathrm{IHC}$ was used to assess the proportion of M2-like macrophages surrounding tumor tissues (Fig. 4C), and the results demonstrated the number of M2 macrophages to be significantly less in RGO-SMEDDS-treated mice compared to control. Therefore, RGO-SMEDDS improved the immunosuppressive microenvironment by inhibiting the M2-type differentiation of macrophages.

\section{EGFR may be a potential target of Oridonin.}


In order to explore the anti-tumor mechanism of Oridonin, the 3D structure (obtained from PubChem) was used to predict Oridonin targets by use of Pharmmapper (Fig. 5A). GO enrichment analysis of predicted Oridonin targets were mainly tyrosine kinases, which are closely related to tumor occurrence and development (Fig. 5B, C). AutoDock 1.1.2 software was used to simulate molecular docking between Oridonin and the tyrosine kinase receptor, EGFR (Fig. 5D-F). We found Oridonin to form hydrogen bonds with the amino acid residue, Arg841, and to form hydrophobic interactions with amino acid residue Cys797, Val726, and Leu844. Moreover, Oridonin fit well within the activation cavity of EGFR (docking score $-7.2 \mathrm{kcal} / \mathrm{mol}$ ). These results suggest Oridonin to be a potential inhibitor of EGFR.

\section{RGO-SMEDDS suppresses HCC stemness via the EGFR/AKT and GSK3a/ $\beta$ signaling pathways.}

IHC staining demonstrated EGFR and P-EGFR levels to be significantly lower than control with RGO treatment (Fig. 6A).By WB, EGFR and P-EGFR were detected in HCC cells treated with RGO-SMEDDS and EGF(Fig. 6B). The results showed that RGO-SMEDDS effectively inhibited EGFR phosphorylation induced by EGF. In order to investigate the anti-tumor mechanism of RGO-SMEDDS, we detected downstream molecules of the EGFR signaling by WB (Fig. 6C). With RGO-SMEDDS treatment, the levels of AKT/p70S6K and GSK3a/ $\beta$ were significantly reduced compared to control group, suggesting that RGOSMEDDS may exert an anti-tumor effect by inhibition of HCC stemness. A sphere formation assay was used to confirm this speculation. Results demonstrated that RGO-SMEDDS treatment significantly reduced the number of spheres compared to control (Fig. 6D, E). In addition, the results of qRT-PCR showed that RGO-SMEDDS down-regulated mRNA expression of stemness marker both in HCC cells and tumor tissues (Fig. 6G). By WB, similar results were obtained (Fig. 6F). These results demonstrated RGOSMEDDS to attenuate HCC stemness by suppressing the EGFR/AKT and GSK3a/ $\beta$ signaling pathways.

\section{RGO-SMEDDS is a safe and effective anti-tumor drug.}

Within 14 days after administration, the mice in the $1000 \mathrm{mg} / \mathrm{kg}$ group showed sluggish activity within 2-3 hours after administration and the other group $(0,100,200, \mathrm{mg} / \mathrm{kg})$ were in good condition without significant weight loss. To assess RGO-SMEDDS toxicity, HE staining was performed on tissue sections of major organs. No histopathological changes were found in the brain, gastric, colon, heart, liver, spleen, lung and kidney tissues, indicating that RGO-SMEDDS has a relatively low degree of toxicity (sFig. 2). Since no half-lethal dose of RGO-SMEDDS was found, we performed an LD50 test with the maximum dose $(1,000 \mathrm{mg} / \mathrm{kg})$. The results of HE staining were shown in Fig. 7 and are consistent with previous experiments.

\section{Discussion}

HCC is one of the most aggressive cancers with an extremely low survival rate and a high risk for metastasis. Diagnosis is frequently conducted during late stages of the disease when treatments is extremely poor [44]. The molecular mechanisms involved in tumorigenesis are multi-facet and complex with tolerance to monotherapies easily generated [45]. Hence, combinatorial therapies are employed to maximize treatment efficacy [8]. Herein, RGO-SMEDDS was shown to have superior therapeutic efficacy 
against HCC both in vitro and in vivo. RGO-SMEDDS acts by targeting multiple anti-tumor mechanisms: $\mathbb{Z}$ altering the immune-tolerant tumor microenvironment by reducing the production of immune-suppressive cytokines, such as IL-4, IL-10, IL-13, and M-CSF1; $₫$ producing an anti-angiogenic effect by reducing VEGF and VEGFR expression; $₫$ down- regulating cell stemness by inhibiting the EGFR signaling pathway ( Fig. 8). Based on various studies, anti-angiogenic drugs combined with immunotherapy are a promising cancer treatment strategy [46-48]. Anti-angiogenic drugs may recover the host's potent anti-tumor immune response by interfering with multiple steps of the cancer immunity cycle. These include promotion of antigen presentation and lymphocyte infiltration [49-52], activation of cytotoxic CD8 + T cells, and immune suppression reduction [53-55]. On the other hand, immunotherapy reduces tumor vascular density, improves vessel perfusion, alleviates tumor tissue hypoxia, and promotes normalization of the tumor vasculature [55-57]. In addition, immunosuppressive microenvironments weaken the effect of pro-apoptotic drugs through adaptive resistance, and an abnormal blood supply affects the entry of pro-apoptotic drugs into tumor tissue. As such, immune and angiogenesis therapies are also indispensable when growth inhibitors are used for treatment [56-59]. These anti-tumor effects suggest a role for RGO-SMEDDS as a novel and effective candidate for liver cancer treatment.

Tumor cells typically induce an immune-tolerant tumor microenvironment by secretion of suppressive molecules such as IL-10, TGF- $\beta$, prostaglandin E2, and VEGF [60]. In addition, tumor cells have been reported to induce M2-like differentiation of macrophages to reduce immune attack by secretion of the cytokines IL-4, IL-10, and M-CSF [61-63]. Macrophages, called Kuper cells in the liver, inhibit the early development of hepatocellular carcinoma; However, during tumor progression, M1 switch to M2, leading to adaptive immune system suppression and tumor support [64, 65]. Furthermore, M2 macrophages promote tumor progression and metastasis, and TAM infiltration is associated with poor prognosis in liver cancers [66]. In this study, RGO-SMEDDS treatment inhibited HCC secretion of the immunosuppressive cytokines IL-4, IL-10, IL-13, and M-CSF. This inhibition effectively blocked the induction of M2-like macrophage differentiation by HCC cells, resulting in improved anti-tumor immune function.

EGFR, a member of receptor tyrosine kinases (RTKs) family, has emerged as an important therapeutic target [67]. Overexpression of EGFR is frequently observed in HCC, suggesting that EGFR might play an important role In HCC pathogenesis and treatment [68]. In this study, we predicted, by use of the PharmMapper website and molecular docking assay, that EGFR may be the potential target of Oridonin. By WB, RGO-SMEDDS was shown to inhibit the phosphorylation of EGFR, suggesting that RGO-SMEDDS might suppress the downstream signaling pathways of EGFR. Previous studies have shown that tumor cells expressing low levels of EGFR and laryngeal squamous cell carcinomas cells with EGFR knockdown are less sensitive to Oridonin [69]. In addition, Oridonin has been shown to inhibit EGFR-mediated $\mathrm{PI} 3 \mathrm{~K} / \mathrm{AKT}$ and Ras/Raf/MEK/ERK pathways, which is consistent with our results [70, 71]. Glycogen synthase kinase-3 beta (GSK-3 $\beta$ ) and mammalian targets of rapamycin (mTOR), major targets correlated with the PI3K/AKT pathway, are involved in the regulation of tumor cell stemness. Therefore, we analyzed the effect of RGO-SMEDDS on the stemness of HCC. Results showed that RGO-SMEDDS inhibited tumor cell sphere formation and down-regulated the expression levels of stemness markers. Cancer stem cells 
(CSCs) have been shown as the main cause of treatment resistance and cancer recurrence due to pluripotency and self-renewal ability [72]. Further, CSCs have been implicated in cancer cell growth, invasion/metastasis, vasculogenesis, and immunosuppression [73]. Hence, RGO-SMEDDS inhibition of tumor cell stemness may benefit patients with liver cancer.

Combinatorial treatment of cancer has provoked widespread concern because of the associated challenges such as plasma instability, low bioavailability, and systemic toxicity [3]. In this study, in addition to its excellent anti-tumor effects, RGO-SMEDDS has been shown to be extremely safe in acute toxicity tests. When the mice were given a large dose of $1000 \mathrm{mg} / \mathrm{kg}$, no pathological changes occurred in important organs. This dose was 100 times that of the actual clinical dosage, indicating that RGOSMEDDS has no obvious toxic side effects. The safety of RGO-SMEDDS may be attributed to the following: $\otimes R g 3, G L P$, and Oridonin are less toxic as natural drugs [74, 75]; $₫$ Synergistic effects among drugs reduce the unnecessary drug amount while still achieved equal efficiency [6, 8]; $₫$ SMEDDS improve the bioavailability of drugs $[34,76]$.

\section{Conclusion}

In summary, we investigated the functional role and molecular mechanism of RGO-SMEDDS as a potential treatment for HCC. The results showed RGO-SMEDDS effectively played an anti-tumor role both in vivo and in vitro, with good safety. RGO-SMEDDS promoted apoptosis, induced cycle arrest, downregulated stemness, resisted angiogenesis, inhibited the release of immunosuppressive cytokines, and reduce the proportion of M2-polarized macrophages. In addition, RGO-SMEDDS inhibited the phosphorylation of EGFR and the activation of downstream AKT/ GSK-3a/ $\beta$ signaling pathways. These results suggested that RGO-SMEDDS may be a potentially effective treatment strategy for improving the survival of HCC patients.

\section{Abbreviations}

HCC \hepatocellular carcinoma; RGO: Rg3, Ganoderma lucidum polysaccharide, and Oridonin; SMEDDS: self-microemulsifying drug delivery system; RT-PCR: real Time-polymerase chain reaction; EGFR: epidermal growth factor receptor; GSK-3a/ $\beta$ : glycogen synthase kinase-3 alpha/beta; TME: tumor microenvironment ; GLP: Ganoderma lucidum polysaccharides; VEGF: vascular endothelial growth factor receptor; VEGFR: vascular endothelial growth factor receptor; FCM: flow cytometry; PI: propidium iodide; V-FITC: V-fluorescein isothiocyanate; WB: western blot; LD50: 50\% lethal dose; IC50: 50\% inhibitive concentration; IHC: immunohistochemistry; Arg1: arginase 1; M-CSF1: Colony Stimulating Factor 1; IL10: interleukin 10.

\section{Declarations}

Acknowledgments 
We thank the other partners of our laboratory for help suggestions

\section{Author contributions}

TX: conception and design. TX, SH: performed majority of experiments. TX, SH,ST,JM,QX,YW,: performed experiments and analyzed data. JC,HC,JC: Preparation of RGO-SMEDD.TX,SH: drafted the manuscript. TX『SH: reviewed data and manuscript. TX, SH,XL,KX,YN: reviewed data and finalized the manuscript. All authors reviewed and approved the final version.

\section{Funding}

This study was supported by National Natural Science Foundation of China (\#81872380, \#81572769), Natural Science Foundation of Chongqing (20200911-2 ), TCM technology Innovation Project of Chongqing (2020ZY013779).

\section{Availability of data and materials}

The datasets used and/or analyzed during the current study are available from the corresponding author on reasonable request.

\section{Ethics approval and consent to participate}

Informed consent was obtained from each participant included in the study. This research was approved by the Institutional Ethics Committees of the First Affiliated Hospital of Chongqing Medical University (Approval notice: \# 2018-77) and conformed to the tenets of the Declaration of Helsinki.

\section{Consent for publication}

Not applicable.

\section{Competing interests}

All authors declare that they have no competing interests.

\section{References}

1. Siegel Rebecca L, Miller Kimberly D. Jemal Ahmedin,Cancer statistics, 2019.[J].CA. Cancer J Clin. 2019;69:7-34.

2. Yang JD, et al. A global view of hepatocellular carcinoma: trends, risk, prevention and management. Nature Reviews Gastroenterology Hepatology. 2019;16(10):589-604.

3. Wang J, et al. Salinomycin-Loaded Small-Molecule Nanoprodrugs Enhance Anticancer Activity in Hepatocellular Carcinoma. Int J Nanomedicine. 2020;15:6839-54.

4. Zhang Y, et al. All-trans retinoic acid potentiates the chemotherapeutic effect of cisplatin by inducing differentiation of tumor initiating cells in liver cancer. J Hepatol. 2013;59(6):1255-63. 
5. Hidalgo $M$, et al. Addressing the challenges of pancreatic cancer: Future directions for improving outcomes. Pancreatology. 2015;15(1):8-18.

6. Parhi P, Mohanty C, Sahoo SK. Nanotechnology-based combinational drug delivery: an emerging approach for cancer therapy. Drug Discov Today. 2012;17(17-18):1044-52.

7. Kobayashi Y, Lim S, Yamaguchi H. Oncogenic signaling pathways associated with immune evasion and resistance to immune checkpoint inhibitors in cancer. Semin Cancer Biol. 2020;65:51-64.

8. Gregory JV, et al., Programmable Delivery of Synergistic Cancer Drug Combinations Using Bicompartmental Nanoparticles. Advanced Healthcare Materials, 2020: p. 2000564.

9. Yap TA, Omlin A, de Bono JS. Development of Therapeutic Combinations Targeting Major Cancer Signaling Pathways. Journal of clinical oncology. 2013;31(12):1592-605.

10. André $F$, et al., Comparative genomic hybridisation array and DNA sequencing to direct treatment of metastatic breast cancer: a multicentre, prospective trial (SAFIR01/UNICANCER). The lancet oncology, 2014. 15(3): p. 267-274.

11. Gao X, et al. Targeting nanoparticles for diagnosis and therapy of bone tumors: Opportunities and challenges. Biomaterials. 2021;265:120404.

12. McGranahan N, et al. Allele-Specific HLA Loss and Immune Escape in Lung Cancer Evolution. Cell. 2017;171(6):1259-71.e11.

13. Tran E, et al. T-Cell Transfer Therapy Targeting Mutant KRAS in Cancer. N Engl J Med. 2016;375(23):2255-62.

14. Chowell D, et al. Patient HLA class I genotype influences cancer response to checkpoint blockade immunotherapy. Science. 2018;359(6375):582-7.

15. Böttcher JP, et al. NK Cells Stimulate Recruitment of $\mathrm{CDC1}$ into the Tumor Microenvironment Promoting Cancer Immune Control. Cell. 2018;172(5):1022-37.e14.

16. Böger $C$, et al. The novel negative checkpoint regulator VISTA is expressed in gastric carcinoma and associated with PD-L1/PD-1: A future perspective for a combined gastric cancer therapy? Oncoimmunology. 2017;6(4):e1293215.

17. Snyder A, et al. Genetic Basis for Clinical Response to CTLA-4 Blockade in Melanoma. N Engl J Med. 2014;371(23):2189-99.

18. Topalian SL, Drake CG, Pardoll DM. Targeting the PD-1/B7-H1(PD-L1) pathway to activate anti-tumor immunity. Curr Opin Immunol. 2012;24(2):207-12.

19. Mantovani A, et al. Tumour-associated macrophages as treatment targets in oncology. Nature Reviews Clinical Oncology. 2017;14(7):399-416.

20. Sakaguchi M. S100-SPECT uncovers cellular and molecular events of pre-metastatic niche formation and following organ-specific cancer metastasis. Theranostics. 2017;7(10):2649-51.

21. D S. and H. S, Ganoderma lucidum Polysaccharides as An Anti-cancer Agent. Anti-cancer Agents Med Chem. 2018;18(5):667-74. 
22. Wang Y, Fan X, Wu X. Ganoderma lucidum polysaccharide (GLP) enhances antitumor immune response by regulating differentiation and inhibition of MDSCs via a CARD9-NF-KB-IDO pathway. Bioscience Reports, 2020. 40(6).

23. Wang P, Zhu X, Lin Z. Antitumor and Immunomodulatory Effects of Polysaccharides from BrokenSpore of Ganoderma lucidum. Frontiers in Pharmacology, 2012. 3.

24. Kladar Nebojša V,Gavarić Neda S. Božin Biljana N,Ganoderma: insights into anticancer effects.[J]. Eur J Cancer Prev. 2016;25:462-71.

25. Cao X, et al. Upregulation of VEGF-A and CD24 gene expression by the tGLI1 transcription factor contributes to the aggressive behavior of breast cancer cells. Oncogene. 2012;31(1):104-15.

26. Song Yuxiao,Fu Yang,Xie. Qi, et al. Anti-angiogenic Agents in Combination With Immune Checkpoint Inhibitors: A Promising Strategy for Cancer Treatment[J]Front Immunol, 2020, 11: 1956.

27. Qiao L, et al. Advanced research on vasculogenic mimicry in cancer. J Cell Mol Med. 2015;19(2):315-26.

28. Sun M, et al. Anticancer effects of ginsenoside Rg3 (Review). Int J Mol Med. 2017;39(3):507-18.

29. Hu S, et al., Ginsenoside Rg3 Prolongs Survival of the Orthotopic Hepatocellular Carcinoma Model by Inducing Apoptosis and Inhibiting Angiogenesis. Analytical Cellular Pathology, 2019. 2019: p. 1-7.

30. Chen QJ, Zhang MZ, Wang LX. Gensenoside Rg3 inhibits hypoxia-induced VEGF expression in human cancer cells. Cell Physiol Biochem. 2010;26(6):849-58.

31. Kim JW, et al. Ginsenoside Rg3 attenuates tumor angiogenesis via inhibiting bioactivities of endothelial progenitor cells. Cancer Biol Ther. 2012;13(7):504-15.

32. Liu T, et al. Ginsenoside 20(S)-Rg3 targets HIF-1alpha to block hypoxia-induced epithelialmesenchymal transition in ovarian cancer cells. PLoS One. 2014;9(9):e103887.

33. Li C, et al. Oridonin: An active diterpenoid targeting cell cycle arrest, apoptotic and autophagic pathways for cancer therapeutics. The International Journal of Biochemistry Cell Biology. 2011;43(5):701-4.

34. Zhang Y, et al. Solubility and Bioavailability Enhancement of Oridonin: A Review. Molecules. 2020;25(2):332.

35. Ding Y, et al. Discovery and development of natural product oridonin-inspired anticancer agents. Eur J Med Chem. 2016;122:102-17.

36. Xu J, et al. Therapeutic Potential of Oridonin and Its Analogs: From Anticancer and Antiinflammation to Neuroprotection. Molecules. 2018;23(2):474.

37. Tong XP, et al. Key autophagic targets and relevant small-molecule compounds in cancer therapy. Cell Prolif. 2015;48(1):7-16.

38. Kwan $\mathrm{H}$, et al. The anticancer effect of oridonin is mediated by fatty acid synthase suppression in human colorectal cancer cells. J Gastroenterol. 2013;48(2):182-92.

39. Liu Z, et al. Oridonin: targeting programmed cell death pathways as an anti-tumour agent. Cell Prolif. 2012;45(6):499-507. 
40. Yuan-chao YHWL. Oridonin induces apoptosis and autophagy in murine fibrosarcoma L929 cells partly via NO-ERK-p53 positive-feedback loop signaling pathway. Acta pharmacologica Sinica. 2012;33(8):1055-61.

41. Laffleur, Flavia. Keckeis Valérie,Advances in drug delivery systems: Work in progress still needed?[J]. Int J Pharm. 2020;590:119912.

42. Nardin, Isabelle. Köllner Saskia,Successful development of oral SEDDS: screening of excipients from the industrial point of view.[J]. Adv Drug Deliv Rev. 2019;142:128-40.

43. Shao B, et al., The 3p14.2 tumour suppressor ADAMTS9 is inactivated by promoter CpG methylation and inhibits tumour cell growth in breast cancer. Journal of Cellular and Molecular Medicine, 2017.

44. Wu CX, et al. Blocking CDK1/PDK1/ $\beta$-Catenin signaling by CDK1 inhibitor R03306 increased the efficacy of sorafenib treatment by targeting cancer stem cells in a preclinical model of hepatocellular carcinoma. Theranostics. 2018;8(14):3737-50.

45. Mazzanti R, Gramantieri L, Bolondi L. Hepatocellular carcinoma: Epidemiology and clinical aspects. Mol Aspects Med. 2008;29(1-2):130-43.

46. Song Y, et al., Anti-angiogenic Agents in Combination With Immune Checkpoint Inhibitors: A Promising Strategy for Cancer Treatment. Front Immunol, 2020. 11: p. 1956.

47. Raybould Alison L. Sanoff Hanna,Combination Antiangiogenic and Immunotherapy for Advanced Hepatocellular Carcinoma: Evidence to Date.[. J]J Hepatocell Carcinoma. 2020;7:133-42.

48. Yi M, et al., Synergistic effect of immune checkpoint blockade and anti-angiogenesis in cancer treatment. Molecular Cancer, 2019. 18(1).

49. Long J, et al., VEGF Impairs the Motility and Immune Function of Human Mature Dendritic Cells throughVEGFR2-RhoA-Cofilin1 Pathway. Cancer Science, 2019.

50. Aggarwal V, et al. Role of Reactive Oxygen Species in Cancer Progression: Molecular Mechanisms and Recent Advancements. Biomolecules. 2019;9(11):735.

51. Malo CS, et al., Immunomodulation Mediated by Anti-angiogenic Therapy Improves CD8 T Cell Immunity Against Experimental Glioma. Frontiers in Oncology, 2018. 8.

52. Delfortrie S, et al. Egfl7 Promotes Tumor Escape from Immunity by Repressing Endothelial Cell Activation. Can Res. 2011;71(23):7176-86.

53. Voron T, et al. VEGF-A modulates expression of inhibitory checkpoints on CD8 + T cells in tumors. J Exp Med. 2015;212(2):139-48.

54. De Sanctis F, et al. The dark side of tumor-associated endothelial cells. Semin Immunol. 2018;35:3547.

55. Voron T, et al. VEGF-A modulates expression of inhibitory checkpoints on CD8 + T cells in tumors. J Exp Med. 2015;212(2):139-48.

56. Xiang D, et al. Oncofetal HLF transactivates c-Jun to promote hepatocellular carcinoma development and sorafenib resistance. Gut. 2019;68(10):1858-71. 
57. Tovar V, et al. Tumour initiating cells and IGF/FGF signalling contribute to sorafenib resistance in hepatocellular carcinoma. Gut. 2017;66(3):530-40.

58. Da Ros M, et al. Glioblastoma Chemoresistance: The Double Play by Microenvironment and BloodBrain Barrier. Int J Mol Sci. 2018;19(10):2879.

59. Xavier CPR, et al. The Role of Extracellular Vesicles in the Hallmarks of Cancer and Drug Resistance. Cells. 2020;9(5):1141.

60. Gonzalez Hugo,Hagerling Catharina,Werb Zena,Roles of the immune system in cancer: from tumor initiation to metastatic progression.[J].Genes Dev, 2018, 32: 1267-1284.

61. Ham Sunyoung,Lima Luize G, Lek Erica, et al. The Impact of the Cancer Microenvironment on Macrophage Phenotypes[J]Front Immunol. 2020;11:1308.

62. Wang $Q$, et al., Vascular niche IL-6 induces alternative macrophage activation in glioblastoma through HIF-2a. Nature Communications, 2018. 9(1).

63. Kelly $A$, et al. Human monocytes and macrophages regulate immune tolerance via integrin alphavbeta8-mediated TGFbeta activation. J Exp Med. 2018;215(11):2725-36.

64. Shu, Yongheng. Cheng Ping,Targeting tumor-associated macrophages for cancer immunotherapy.[J]. Biochim Biophys Acta Rev Cancer. 2020;1874:188434.

65. Kuang D, et al. Activated monocytes in peritumoral stroma of hepatocellular carcinoma foster immune privilege and disease progression through PD-L1. The Journal of experimental medicine. 2009;206(6):1327-37.

66. De Palma M, Lewis CE. Macrophage regulation of tumor responses to anticancer therapies. Cancer Cell. 2013;23(3):277-86.

67. Sueangoen N, Tantiwetrueangdet A, Panvichian R. HCC-derived EGFR mutants are functioning, EGFdependent, and erlotinib-resistant. Cell \& Bioscience, 2020. 10(1).

68. Ito $\mathrm{Y}$, et al. Expression and clinical significance of erb-B receptor family in hepatocellular carcinoma. British journal of cancer. 2001;84(10):1377-83.

69. Kang N, et al. Cetuximab enhances oridonin-induced apoptosis through mitochondrial pathway and endoplasmic reticulum stress in laryngeal squamous cell carcinoma cells. Toxicol In Vitro. 2020;67:104885.

70. Kadioglu O, et al., Oridonin Targets Multiple Drug-Resistant Tumor Cells as Determined by in Silico and in Vitro Analyses. Frontiers in Pharmacology, 2018. 9.

71. Pi J, et al. GE11 peptide conjugated selenium nanoparticles for EGFR targeted oridonin delivery to achieve enhanced anticancer efficacy by inhibiting EGFR-mediated PI3K/AKT and Ras/Raf/MEK/ERK pathways. Drug Deliv. 2017;24(1):1549-64.

72. Tanabe A, Sahara H. The Metabolic Heterogeneity and Flexibility of Cancer Stem Cells. Cancers. 2020;12(10):2780.

73. Lee H, Hong I. Targeting Liver Cancer Stem Cells: An Alternative Therapeutic Approach for Liver Cancer. Cancers. 2020;12(10):2746. 
74. Ding C, et al. Novel Nitrogen-Enriched Oridonin Analogues with Thiazole-Fused A-Ring: Protecting Group-Free Synthesis, Enhanced Anticancer Profile, and Improved Aqueous Solubility. J Med Chem. 2013;56(12):5048-58.

75. Liu JP, et al. Toxicity of a novel anti-tumor agent 20(S)-ginsenoside Rg3: A 26-week intramuscular repeated administration study in rats. Food Chem Toxicol. 2012;50(10):3388-96.

76. Ali ES, et al., Targeting cancer cells with nanotherapeutics and nanodiagnostics: Current status and future perspectives. Seminars in Cancer Biology, 2020.

\section{Figures}


A
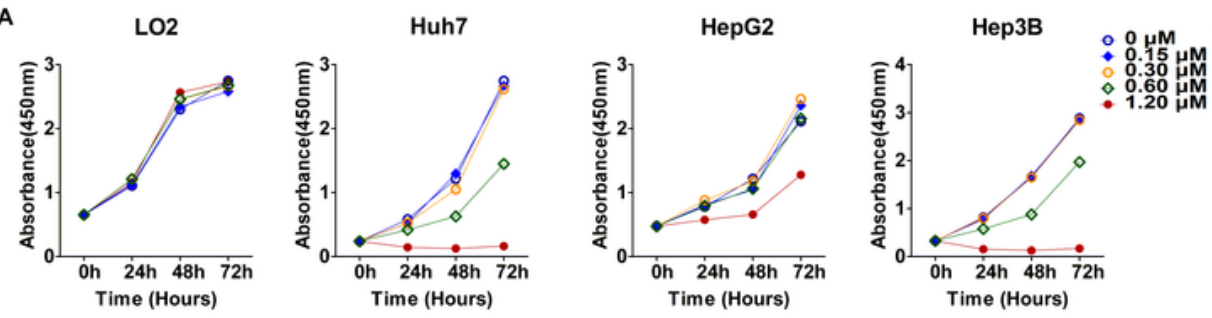

B
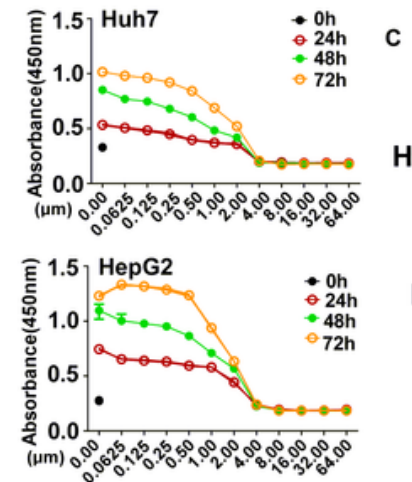

D
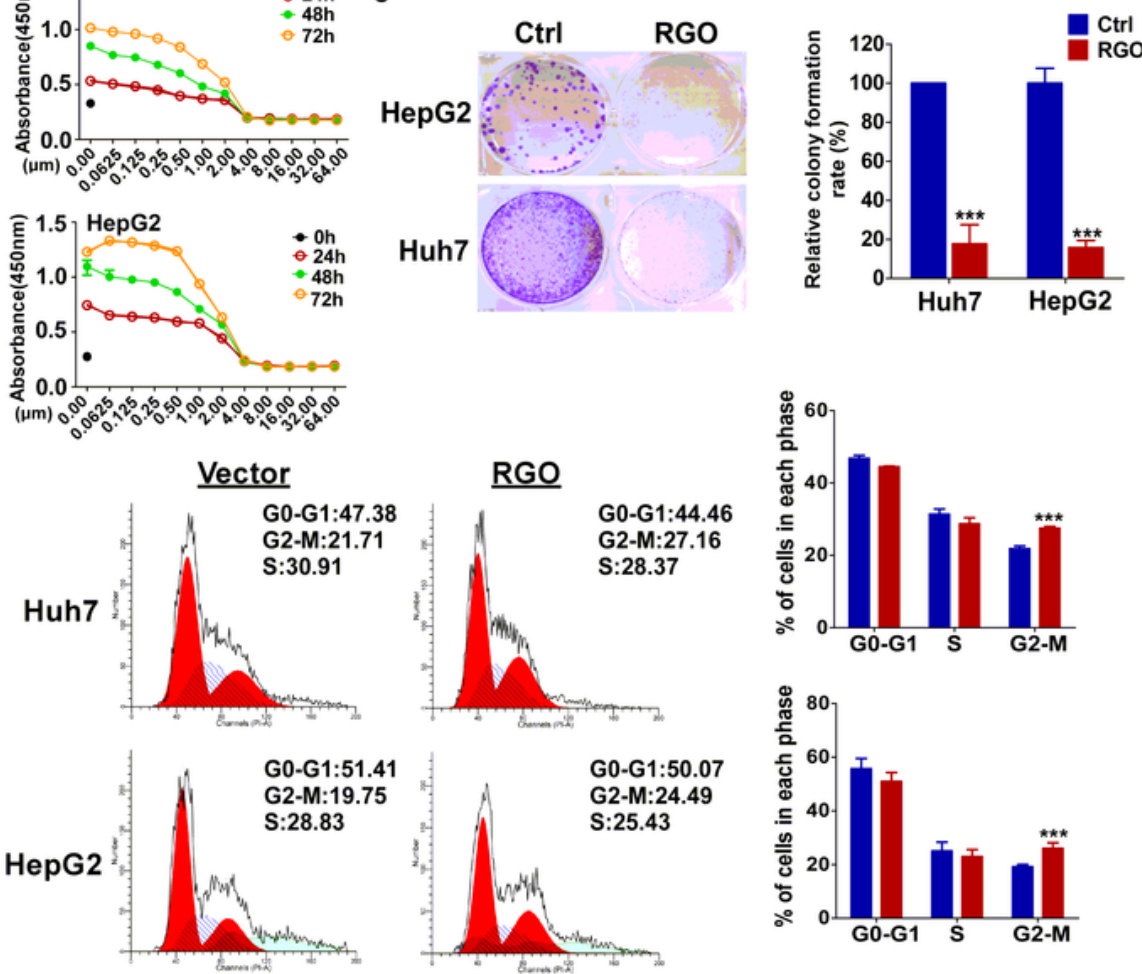

E

Vector
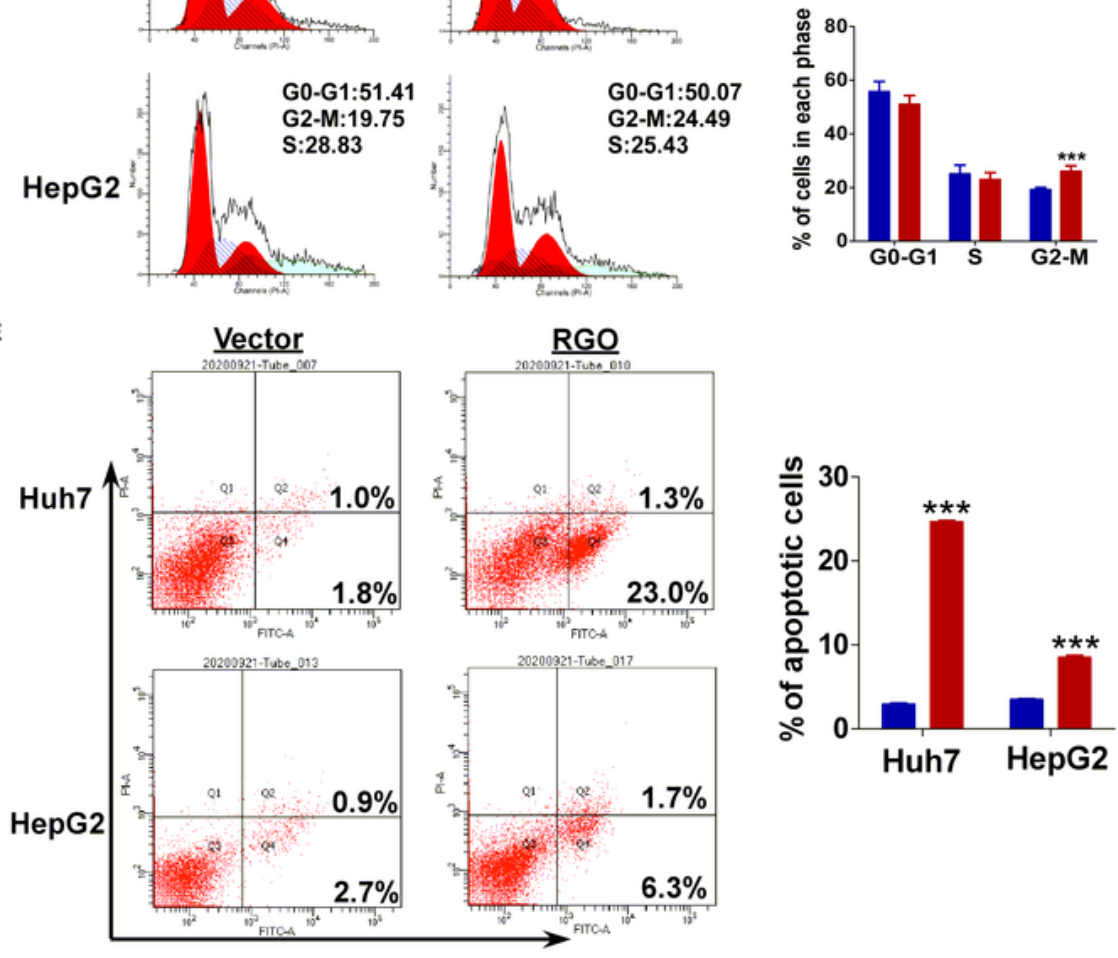

Figure 1

Role of RGO-SMEDDS on hepatoma carcinoma cell growth in vitro (A) Measurement of cell proliferation for $0,0.15,0.30,0.60,1.20$ uM RGO-SMEDDS treated LO2, Huh7, HepG2, and Hep3B cells through CCK-8 assay. (B) IC50 of RGO-SMEDDS against HepG2 and Huh7 cells. (C) Effects of RGO-SMEDDS on the colony formation of HepG2 and Huh7 cells. (D) Cell cycle distribution of RGO-SMEDDS-treated or untreated Huh7 and HepG2 cells determined by flow cytometry. (E) Flow cytometry was used to detect 
apoptosis induced by RGO-SMEDDS. Values are shown as mean \pm standard error from three independent experiments. ${ }^{*} p<0.05,{ }^{* *} p<0.01,{ }^{* * *} p<0.001$.

A
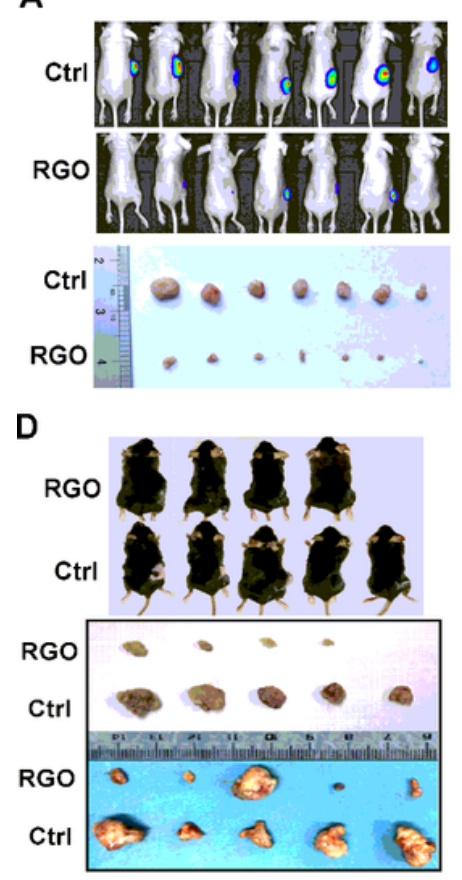

\section{$B$}

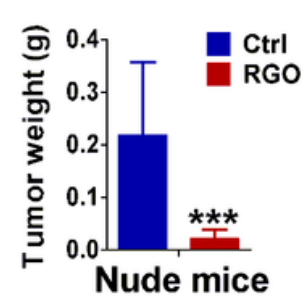

E

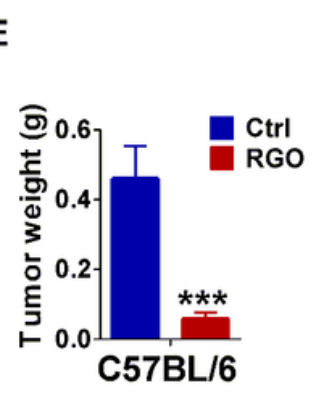

C

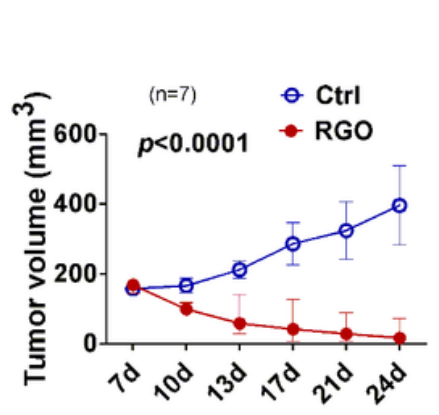

$\mathbf{F}$

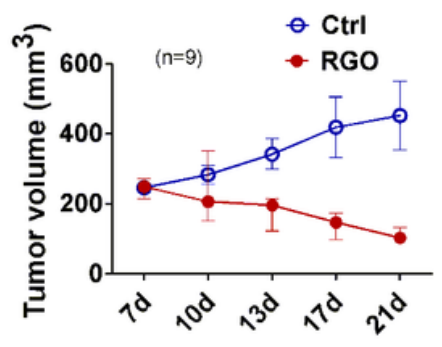

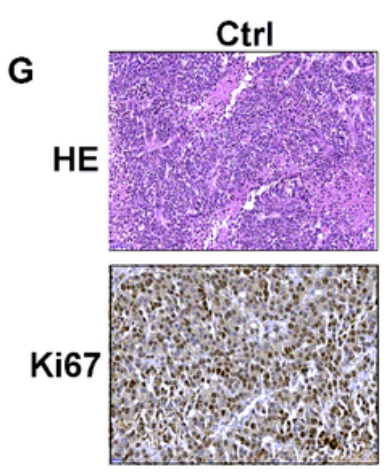
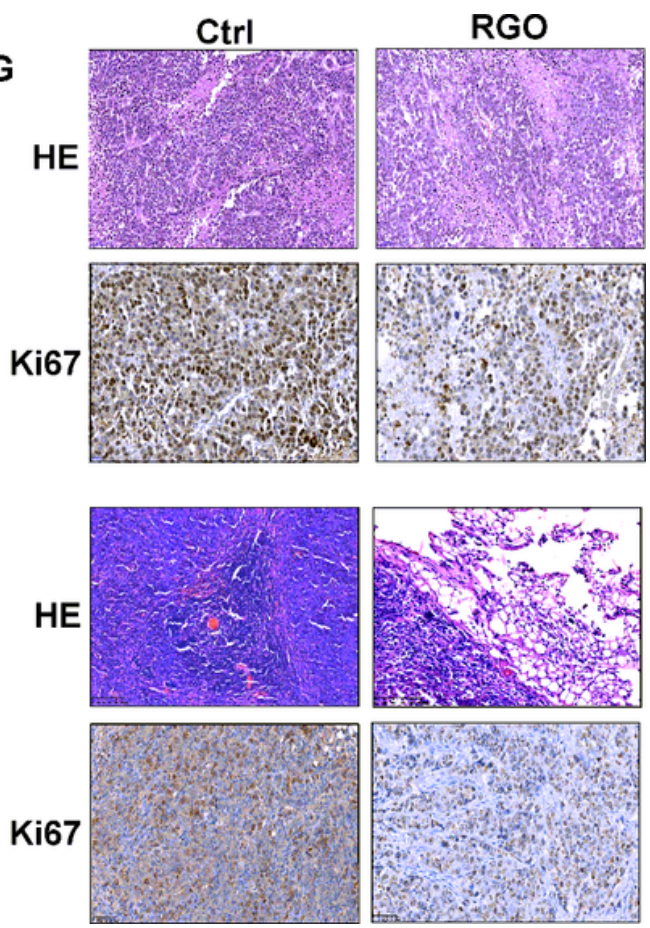

Figure 2

RGO-SMEDDS suppresses xenograft liver tumor growth in vivo (A) Representative images xenograft tumor status assessed by in vivo fluorescence imaging (up) or viewing with naked eye (down). (B-C) Tumor weight by the time of resection and tumor volume growth curve in nude mice. Tumor volume was calculated from tumor length and width, measured every two days. (D) Representative pictures of the xenograft tumors before and after resection in comparison between RGO and the control group. (E-F) Growth curve and weight of xenograft tumors in C57BL/ 6 mice. Tumor volume was calculated from tumor length and width, measured every two days. (G) HE staining and immunohistochemistry $(\mathrm{IHC})$ staining of Ki67 of xenograft tumor tissues from nude mice and C57BL/6 mice. 
A

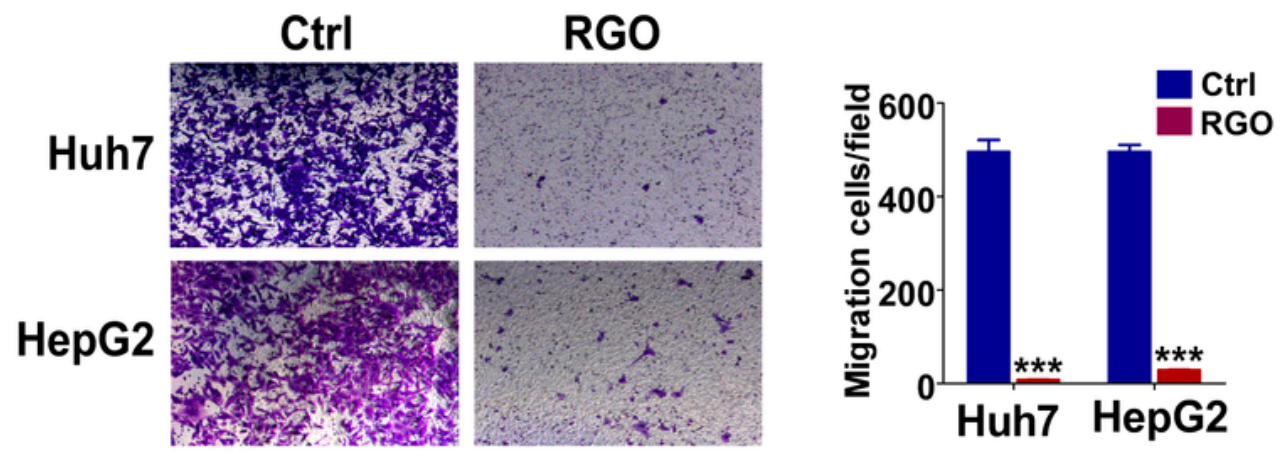

B
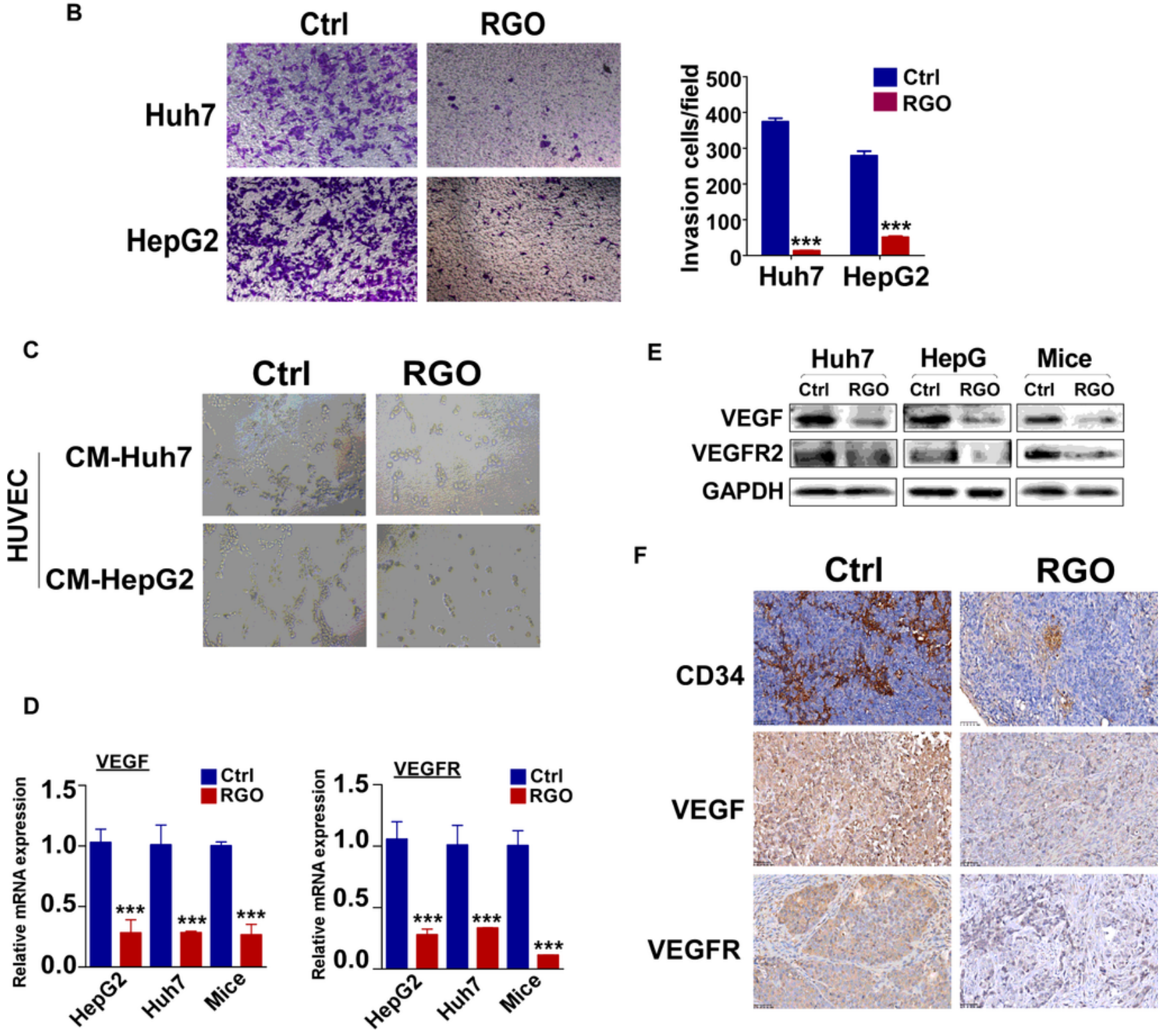

Figure 3

Inhibition of RGO-SMEDDS on migration, invasion and angiogenesis of HCC (A-B) Representative image of Transwell cell migration and invasion assay in Huh7 and HepG2 cells treated with or without RGOSMEDDS, $\times 100$ magnification ( $<$ 0.001). (C) The antiangiogenic ability of RGO-SMEDDS to Huh7 and HepG2 cells detected by tubule formation assay, $\times 100$ magnification. (D) qRT-PCR analyses exhibit the mRNA levels of VEGF and VEGFR in RGO-SMEDDS-treated and untreated HCC. (E) Protein expression 
level of VEGF and VEGFR in HCC treated with RGO-SMEDDS by WB assay. (F) Representative immunohistochemistry staining images of CD34, VEGF and VEGFR in xenograft tumor.

A
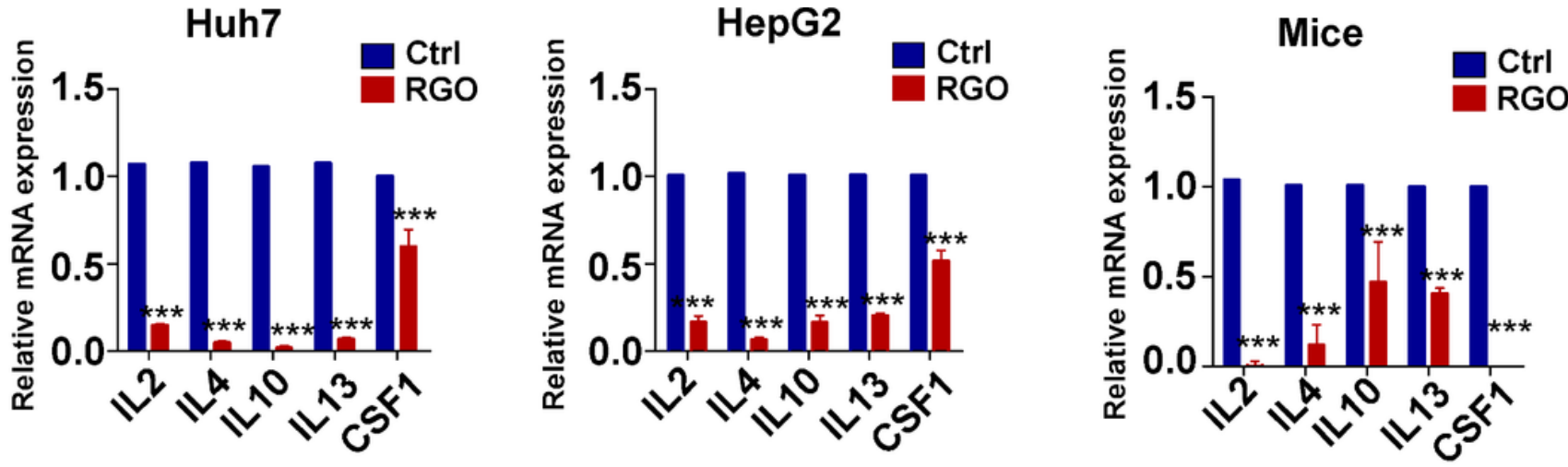

B
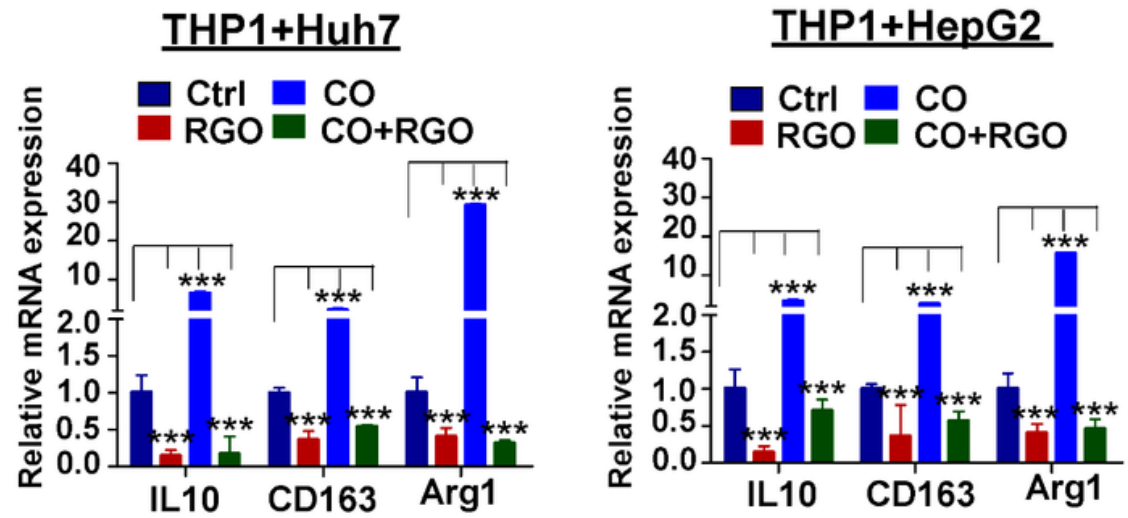

C

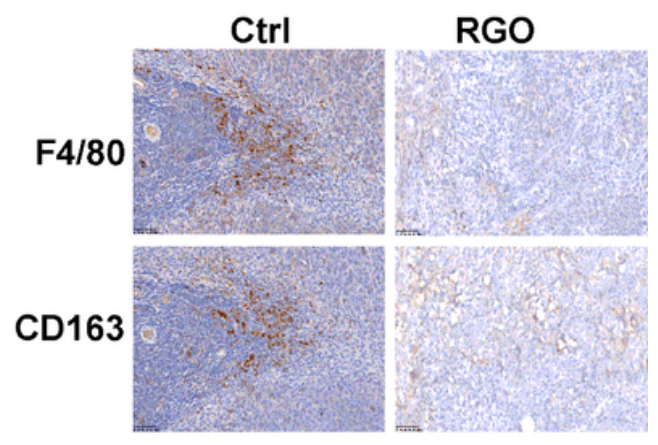

Figure 4

Effects of RGO-SMEDDS on immune microenvironment of hepatocellular carcinoma (A) qRT-PCR analyses was used to detect the mRNA expression level of Immunosuppressive cytokines in RGOSMEDDS-treated and untreated HCC and tumor tissues. (B) the mRNA expression level of of M2 markers for THP-1 cells in the co-culture system with or without RGO-SMEDDS. CO means co-culture .(C) Immunohistochemistry staining of F4/80 and CD163 was used to detect the infiltration of M2 macrophages in tumor tissues of the control group and the RGO-SMEDDS-treated group. 
B

A

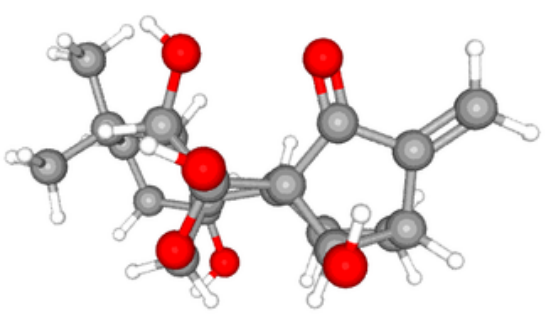

C

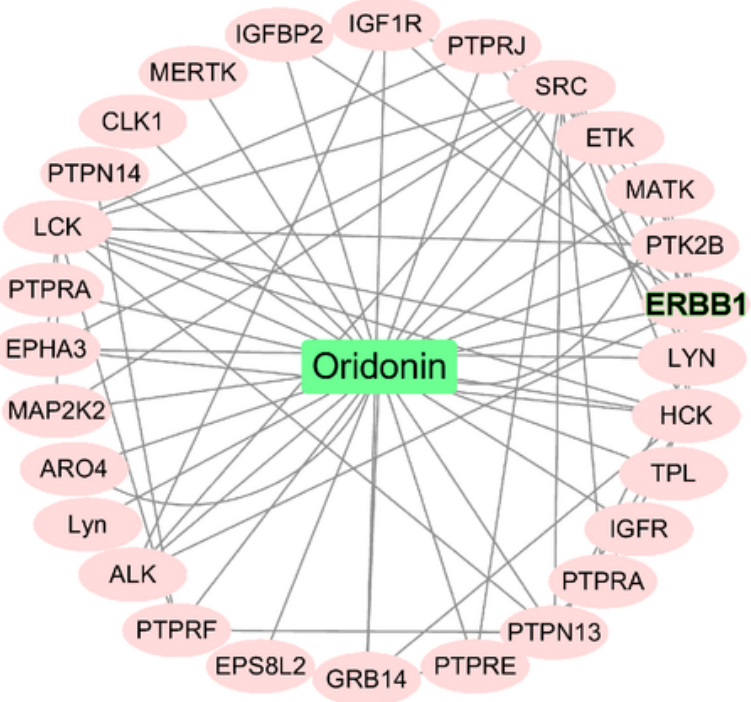

D

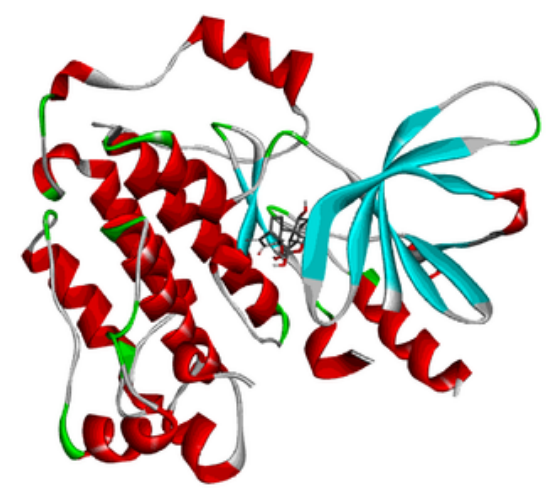

F coenzyme binding

protein serine/threonine

kinase activity amide binding

vitamin binding

metallopeptidase activity

NAD or NADP as acceptor

acting on $\mathrm{CH}-\mathrm{OH}$

group of donors

protein tyrosine kinase activity

damaged DNA binding •

ocarboxylic acid binding -

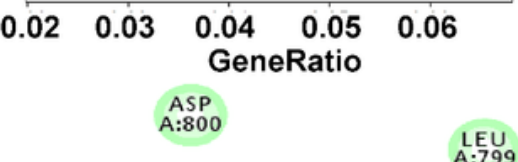

E

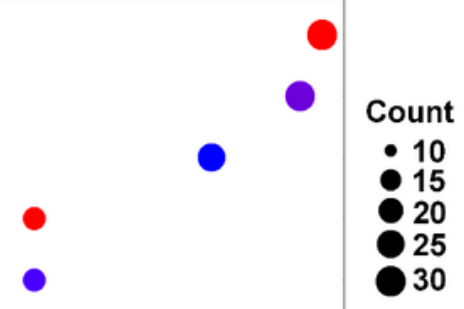

p.adjust

1e-04

2e-04

3e-04

4e-04

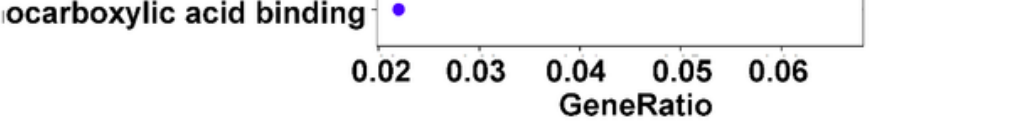

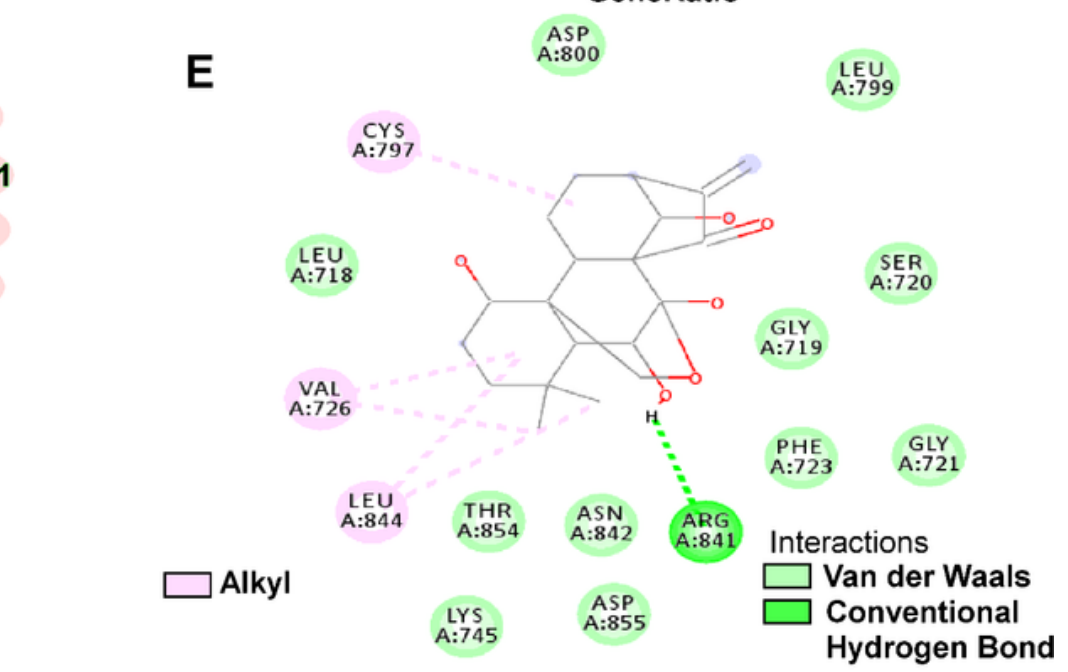

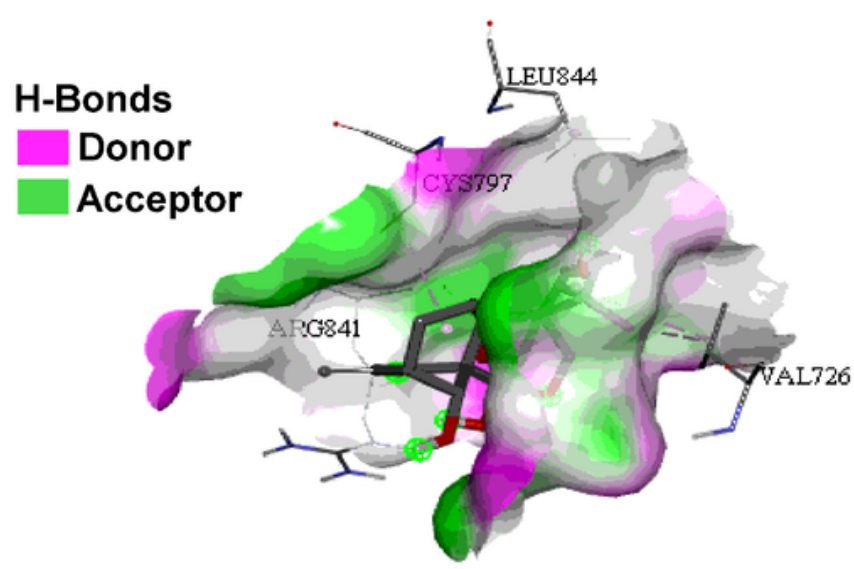

\section{Figure 5}

Target prediction of Oridonin (A) The 3D structure of Oridonin was obtained from the PubChem database.

(B) GO pathway enrichment analysis of the predicted targets of Oridonin was demonstrated by Dotplots.

(C) The PPI network of tyrosine kinase-related protein networks among the predicted targets of Oridonin.

(D) Docking poses in to the pharmacophore of EGFR tyrosine kinase domain (PDB code:1M17 in red 
cartoon representation). Oridonin was represented in gray. (E) The 2-dimensional schematic diagram of interactions of EGFR with Oridonin.(F) The pocket view of EGFR binding with Oridonin.

A

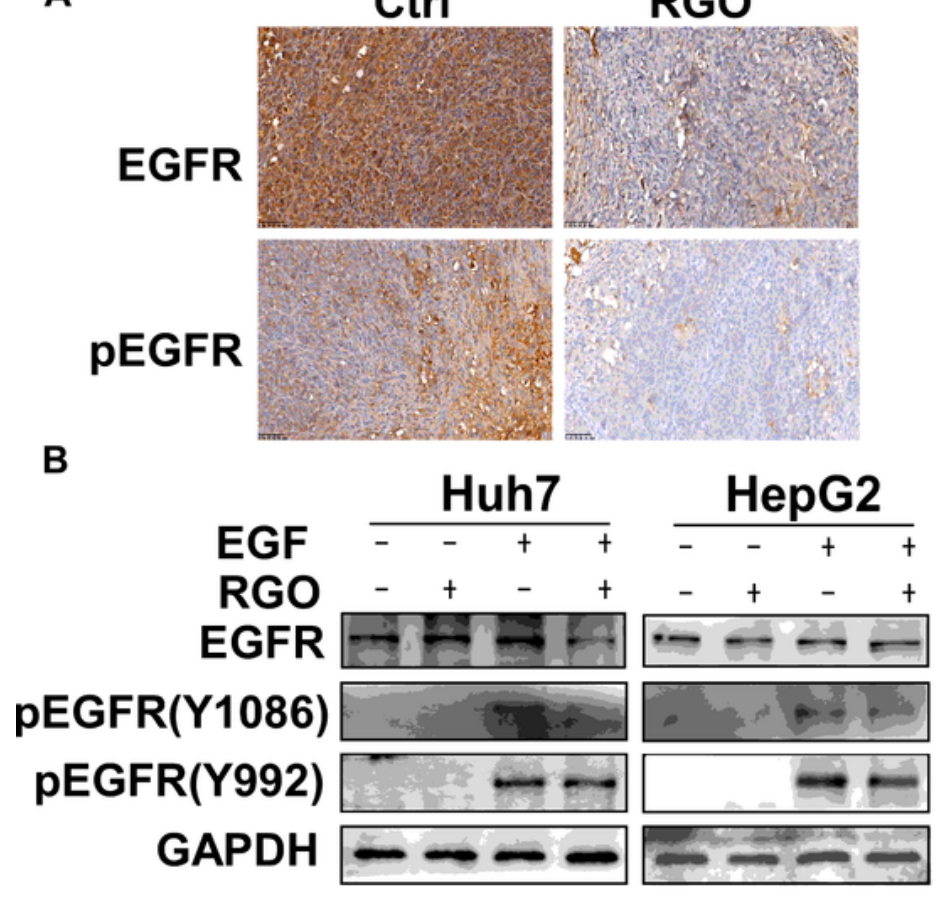

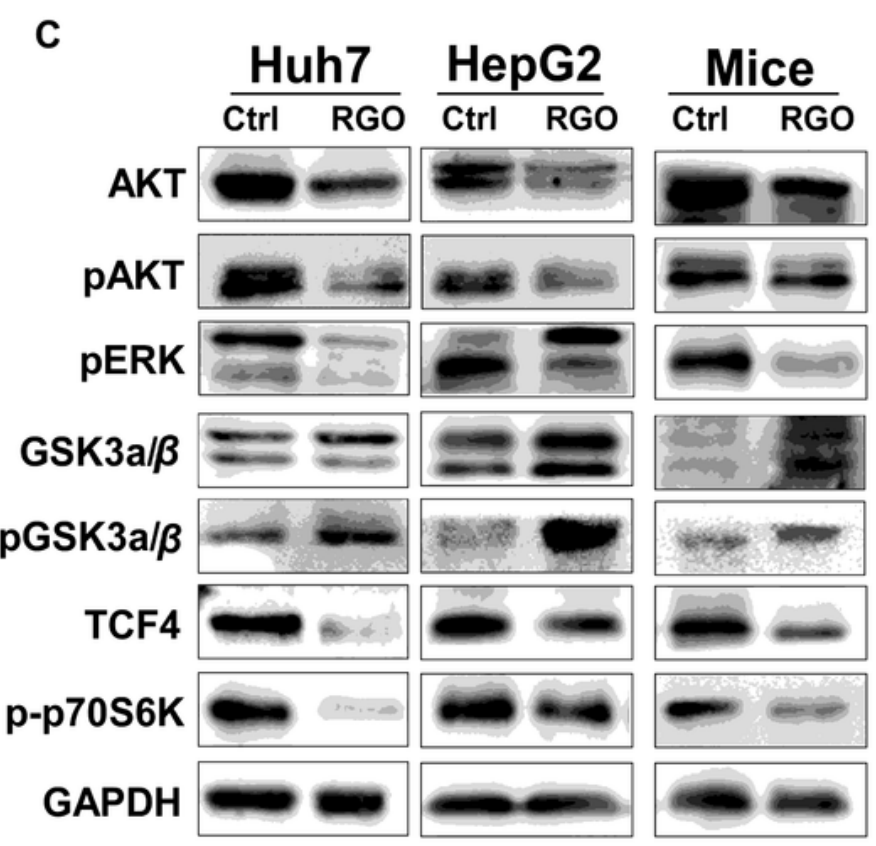

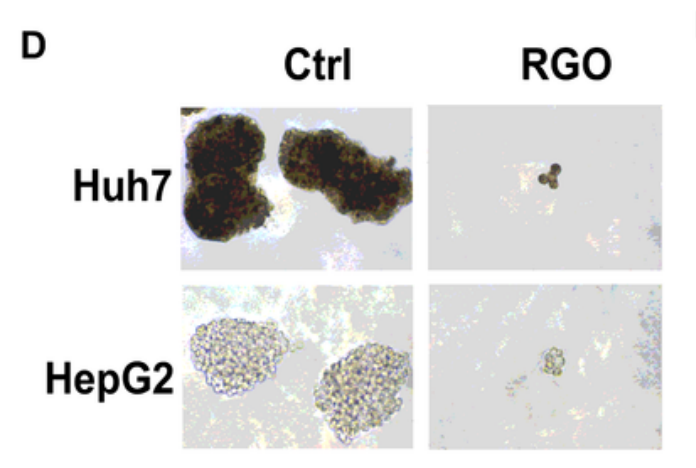

E

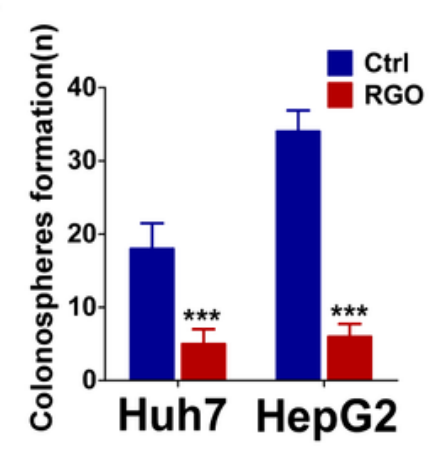

$\mathbf{F}$
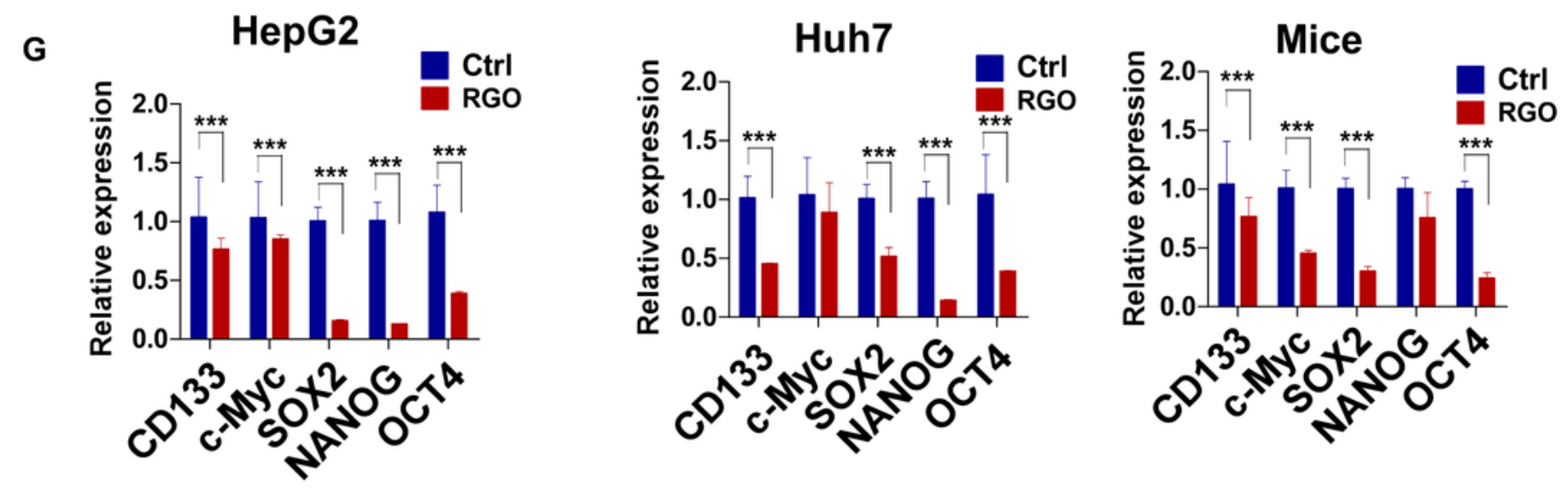

Figure 6

RGO-SMEDDS restrains stemness of HCC through the EGFR/AKT signaling pathway (A) Representative immunohistochemistry staining results of EGFR and P-EGFR in xenografts. (B) The effect of RGOSMEDDS on EGFR phosphorylation in HepG2 and Huh7 cells was detected by WB assay. (C) WB analyses 
was used to detect the downstream molecules expression level of the EGFR signaling pathway in RGOSMEDDS-treated or untreated HepG2 cells, Huh7 cells and tumor tissues. (D-E) Spheroid formation assay showed that RGO-SMEDDS reduced the size of spheres formed by HepG2 and Huh7 cells. ${ }^{* \star}{ }^{2}<0.001$. (F-G) The protein and mRNA expressions level of Stemness markers in RGO-SMEDDS-treated or untreated HepG2 and Huh7 cells determined by WB and qRT-PCR assay.
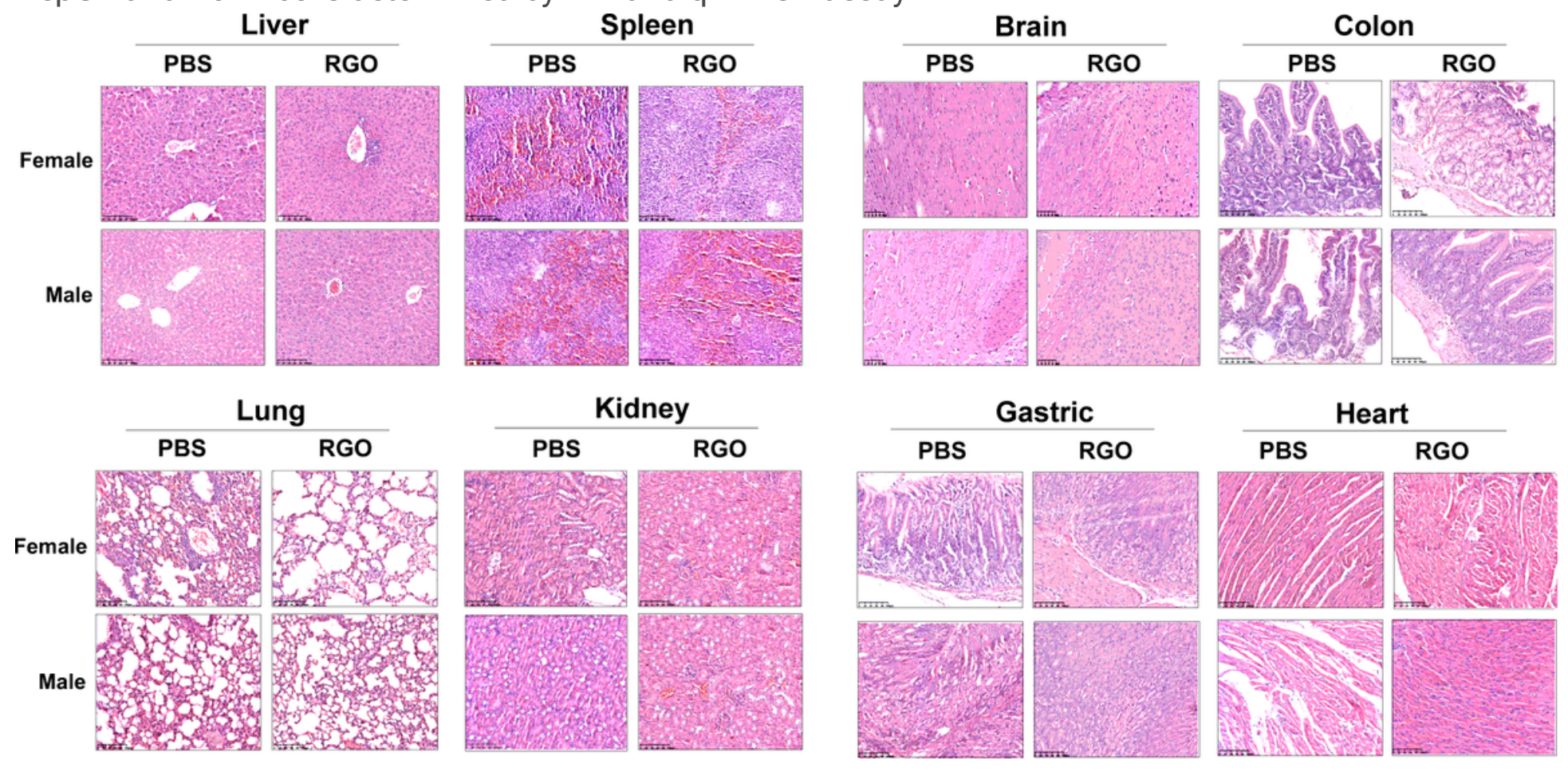

Figure 7

Safety evaluation of RGO-SMEDDS in healthy C57BL/ 6 mice. Representative images of HE staining of major organs (liver, spleen, brain, colon, lung, kidney, gastric, and heart) on day 14 after PBS or $1000 \mathrm{mg} / \mathrm{kg}$ RGO treatment. 


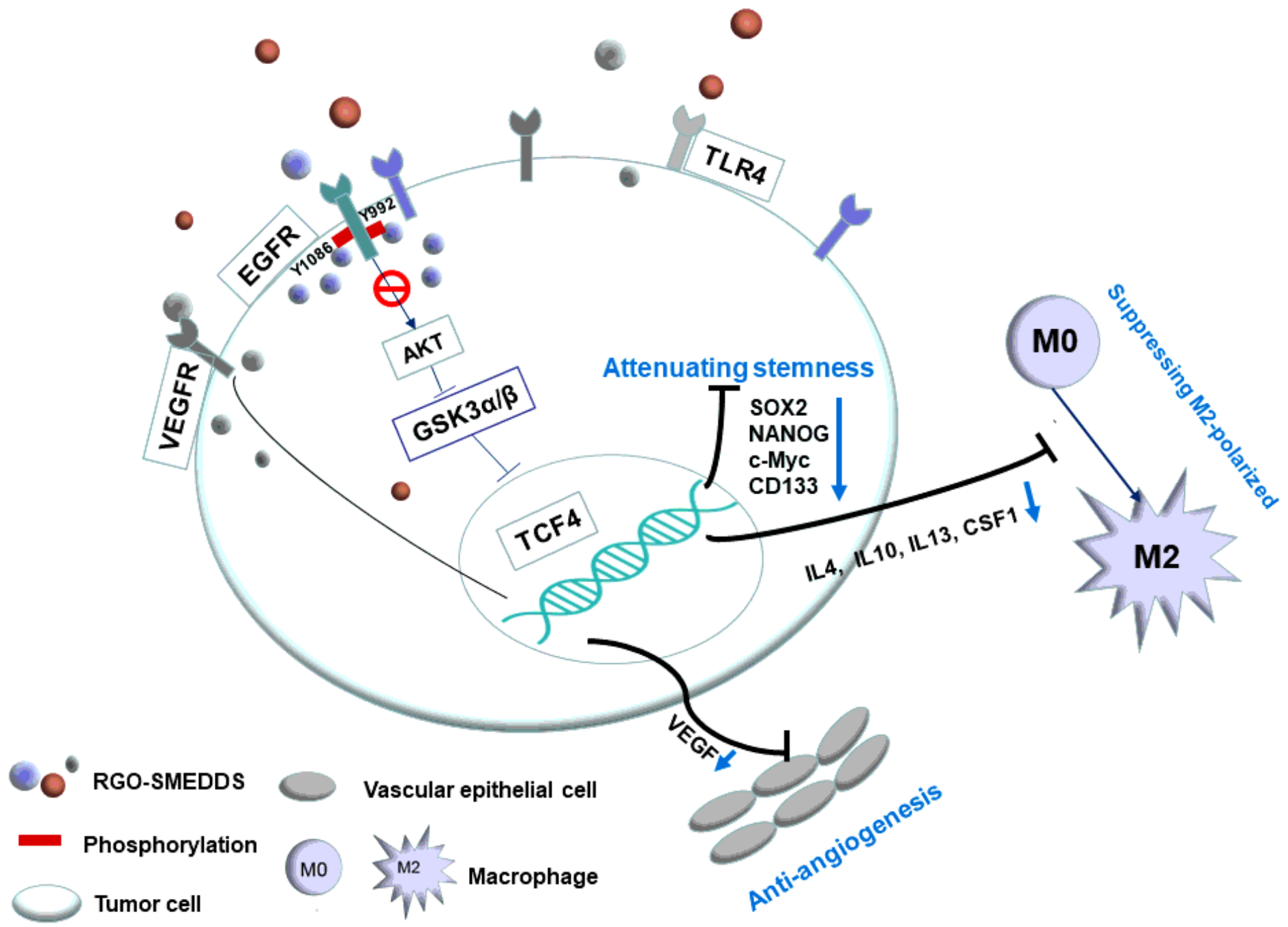

Figure 8

Schematic diagram of the anti-tumor mechanism of RGO-SMEDDS.

\section{Supplementary Files}

This is a list of supplementary files associated with this preprint. Click to download.

- sFig.1.tif

- sFig2.tif 